\title{
COMPARISON OF DUALIZING COMPLEXES
}

\author{
CHANGLONG ZHONG
}

\begin{abstract}
We prove that there is a map from Bloch's cycle complex to Kato's complex of Milnor K-theory, which induces a quasi-isomorphism from cycle complex mod $p^{r}$ to Moser's complex of logarithmic de Rham-Witt sheaves. Next we show that the truncation of Bloch's cycle complex at -3 is quasi-isomorphic to Spiess' dualizing complex. In the end, we prove that a weak form of the Gersten Conjecture implies that Sato's dualizing complex is quasi-isomorphic to Bloch's complex.
\end{abstract}

\section{INTRODUCTION}

Using Lichtenbaum's weight-two motivic complex $\mathbb{Z}(X, 2)$ (a two-term complex derived from relative $\mathrm{K}$-theory), M. Spiess [34] constructed a complex of étale sheaves $\mathcal{K}_{X}$ (Definition 3.1) on arithmetic surfaces $X$ over a Dedekind domain $D$ and used it to prove a duality theorem of constructible sheaves. For $X$ over a perfect field $k$ of characteristic $p>0$, T. Moser [27] studied Gersten complexes of logarithmic de Rham-Witt sheaves $\widetilde{\nu}_{X, r}(n)$ 2.0.6) and showed that, when $k$ is finite, $\widetilde{\nu}_{X, r} \stackrel{\text { def }}{=} \widetilde{\nu}_{X, r}(0)$ is a dualizing complex for constructible $\mathbb{Z} / p^{r}$-sheaves. For regular semi-stable schemes $X$ over certain Dedekind domain $D$ (see Condition 4.1), K. Sato [32] defined certain dualizing complex $\mathfrak{I}_{r}(n)_{X}$ $(0 \leq n \leq \operatorname{dim} X)$ (Definition 4.3) in derived category of étale sheaves and proved a duality theorem for $\mathbb{Z} / p^{r}$-sheaves as well. In more general situations, for instance, schemes over algebraically closed fields, finite fields, local fields and certain Dedekind domains, T. Geisser [9] proved that the complex $\mathbb{Z}_{X}^{c} \stackrel{\text { def }}{=} \mathbb{Z}_{X}^{c}(0)$ of étale sheaves (see (2.0.1) ) is a dualizing complex for constructible sheaves. Here $\mathbb{Z}_{X}^{c}(n)$ is Bloch's cycle complex whose homology defines higher Chow groups [2]. In this paper, we answer the following questions of quasi-isomorphisms of these complexes:

Theorem 1.1 (Theorem 2.16). For $X$ a scheme separated and essentially of finite type over a perfect field $k$ of characteristic $p>0$, and $n \leq d=\operatorname{dim} X$, there is a map

$$
\hat{\psi}: \mathbb{Z}_{X}^{c} / p^{r}(n) \rightarrow \widetilde{\nu}_{X, r}(n)
$$

which induces a quasi-isomorphism. Here $\mathbb{Z}_{X}^{c} / p^{r}(n) \stackrel{\text { def }}{=} \mathbb{Z}_{X}^{c}(n) / p^{r}$.

Theorem 1.2 (Theorem 3.8 and Corollary 3.9). Let $X$ be a surface over a perfect field $k$ or a Dedekind domain $D$ with perfect residue fields. Then $\tau_{\geq-3} \mathbb{Z}_{X}^{c}$ is quasi-isomorphic to $\mathcal{K}_{X}$. Moreover, for any torsion sheaf $\mathcal{F}$,

$$
\operatorname{RHom}_{X}\left(\mathcal{F}, \mathbb{Z}_{X}^{c}\right) \cong \operatorname{RHom}_{X}\left(\mathcal{F}, \mathcal{K}_{X}\right) .
$$

Theorem 1.3 (Theorem 4.8). Let $p$ be a prime number, $X$ be a scheme over a Dedekind domain $D$ which satisfies Condition (4.1) below. Assume that $d=\operatorname{dim} X$, and the conjecture $\mathcal{B}(n)$ with $\mathbb{Z} / p^{r}$-coefficients (Conjecture 2.2) holds for all $x \in X$. Then

$$
\mathfrak{I}_{r}(n)_{X} \stackrel{\cong}{\longrightarrow}\left(\mathbb{Z}_{X}^{c} / p^{r}(d-n)[-2 d]\right) .
$$


Let $X$ be a scheme of dimension $d$ over a perfect field of characteristic $p>0$. Moser's complex $\widetilde{\nu}_{X, r}(n)$ is the Gersten complex of logarithmic de Rham-Witt sheaf $W_{r} \Omega_{X, \log }^{d-n}$, when $X$ is smooth. For general $X, \widetilde{\nu}_{X, r}(n)(X)$ is identified with Kato's complex $C_{X}^{M}(n) / p^{r}$ of Milnor K-groups modulo $p^{r}$ (see Theorem 2.10). To prove Theorem 2.16 first, we show that the niveau filtration of higher Chow groups induces a canonical map

$$
\phi: \mathbb{Z}_{X}^{c}(n)(X) \rightarrow C_{X}^{\mathrm{HC}}(n),
$$

the latter being the global sections of Gersten complex of higher Chow groups (étale sheafified). Then we show that $C_{X}^{\mathrm{HC}}(n)$ is isomorphic to $C_{X}^{\mathrm{M}}(n)$, which provides us a map

$$
\psi: \mathbb{Z}_{X}^{c}(n)(X) \rightarrow C_{X}^{\mathrm{M}}(n) .
$$

When composing with the isomorphism

$$
C_{X}^{M}(n) / p^{r} \cong \widetilde{\nu}_{X, r}(n)(X),
$$

we obtain a map

$$
\hat{\psi}: \mathbb{Z}_{X}^{c} / p^{r}(n)=\mathbb{Z}_{X}^{c}(n) / p^{r} \rightarrow \widetilde{\nu}_{X, r}(n) .
$$

Moreover, using a result of Geisser-Levine [14, Theorem 1.1], we show that $\hat{\psi}$ induces an isomorphism of cohomology groups. Hence we conclude that $\hat{\psi}$ is a quasi-isomorphism. As another application of this method, we show that, for smooth and projective varieties over finite fields, the conjecture A(n) of Geisser (part of Parshin's Conjecture, see [12, Proposition 2.1]) is true, if and only if

$$
\phi_{\mathbb{Q}}: \mathbb{Q}_{X}^{c}(n)(X) \rightarrow C_{X}^{H C}(n) \otimes \mathbb{Q}
$$

is a quasi-isomorphism.

Let $X$ be a two dimensional scheme over a perfect field or a Dedekind domain with perfect residue fields. Spiess' complex $\mathcal{K}_{X}$ is defined by connecting the complex

$$
\bigoplus_{X_{(1)}} i_{x *} \mathbb{G}_{m} \rightarrow \bigoplus_{X_{(0)}} i_{x *} \mathbb{Z}
$$

with Lichtenbaum's weight-two motivic complex $\mathbb{Z}(k(X), 2)$ of the function field $k(X)$ (see [24, Definition 2.1] for $\mathbb{Z}(-, 2)$ ). To compare it with Bloch's complex, we first define an intermediate complex $\mathcal{C}(X)$. Using the niveau spectral sequence, we show that $\mathcal{C}(X)$ is quasi-isomorphic to $\tau_{\geq-3} \mathbb{Z}_{X}^{c}(X)$. Then we show that $\mathcal{C}(X)$ is quasi-isomorphic to $\mathcal{K}_{X}(X)$. A key ingredient in this step is the quasi-isomorphism in [5, §7] between a truncation of Bloch's complex of $k(X)$ and $\mathbb{Z}(k(X), 2)$. Based on the above, we conclude that $\tau_{\geq-3} \mathbb{Z}_{X}^{c}$ is quasi-isomorphic to $\mathcal{K}_{X}$.

Let $X$ be a scheme over a Dedekind domain satisfying Condition 4.1), and $\operatorname{dim} X=d$. Sato's complex $\mathfrak{I}_{r}(n)_{X}$ is defined to be a complex in the derived category of étale sheaves satisfying a distinguished triangle, with the other two terms the logarithmic de RhamWitt sheaf on points of characteristic $p$ and $\mu_{p^{r}}^{\otimes n}$ on the open complement. In order to compare $\mathfrak{I}_{r}(n)_{X}$ with $\mathbb{Z}_{X}^{c} / p^{r}(d-n)[-2 d]$, we show that $\mathbb{Z}_{X}^{c} / p^{r}(d-n)[-2 d]$ satisfies a similar triangle (the localization sequence of Bloch's cycle complex), and that there is a map between the two triangles, which induces isomorphisms on the cohomologies up to degree $n$. The key point is to show that these isomorphisms are compatible. Using the Gersten complexes of $\mu_{p^{r}}^{\otimes d-n}$, cycle complex and logarithmic de Rham-Witt sheaves, we replace maps of cohomology of schemes by those of function fields, whose compatibility is straightforward. Hence the two complexes are quasi-isomorphic up to degree $n$. With the aid of the conjecture $\mathcal{B}(n)$ with $\mathbb{Z} / p^{r}$-coefficients, then they are actually quasi-isomorphic. 
The paper is organized as follows: in Section 2, we recall the definitions of Bloch's cycle complex $\mathbb{Z}_{X}^{c}(n)$, Kato's complex $C_{X}^{M}(n)$ of Milnor K-theory and Moser's complex $\widetilde{\nu}_{r}(n)$, as well as the duality results of Geisser and Moser. We also recall the construction of the niveau spectral sequence of higher Chow groups. For the reader's convenience, we include here a generalization of the Beilinson-Lichtenbaum Conjecture to general schemes and recall Levine's proof of Kummer isomorphism for regular schemes over Dedekind domains [23, Levine, Theorem 12.5], with the assumption of the conjecture $\mathcal{B}(n)$. In Section 3, first we recall Spiess complex $\mathcal{K}_{X}$, his duality results and the definition of Lichtenbaum's weight-two motivic complex $\mathbb{Z}(X, 2)$. Then we define the complex $\mathcal{C}(X)$ and compare it with $\mathbb{Z}_{X}^{c}$ and $\mathcal{K}_{X}$, respectively. In Section 4, we recall Sato's definition of $\mathfrak{I}_{r}(n)_{X}(0 \leq n \leq \operatorname{dim} X)$, and prove Proposition 4.5 and Theorem 4.8 .

\section{TERMINOLOGY}

Throughout this paper, the concepts chain complex and cochain complex are used interchangeably. For instance, if $A$ is a chain complex, we think of it as a cochain complex by letting $A^{n}=A_{-n}$. The convention for shift is: $A[n]^{i}=A^{i+n}$. When talking about truncations, we mean truncations in cohomological degrees.

We use $D$ to denote a Dedekind domain of characteristic 0 with perfect residue fields, and $k$ to denote a perfect field of characteristic $p \geq 0$. There is special assumption for $D$ in Section 4 (Condition 4.1). All the schemes in this paper will be separated and essentially of finite type over $S$ with $S=\operatorname{Spec} k$ or $\operatorname{Spec} D$. By variety we mean schemes separated and of finite type over fields. The dimension of an irreducible $S$-scheme $X$ (or dimension of $X$ over $S$ ) is defined as

$$
\operatorname{dim}_{S} X \stackrel{\text { def }}{=} \operatorname{tr} \cdot \operatorname{deg}(k(X): k(\mathfrak{p}))-\operatorname{ht} \mathfrak{p}+\operatorname{dim} S
$$

where $\mathfrak{p}$ is the image of the generic point of $X$ in $S$, and $k(X)$ is the function field of $X$. If $X$ is of finite type over $S$, then $\operatorname{dim}_{S} X=\operatorname{dim} X$, the Krull dimension of $X$. If $X$ is spectrum of a field of transcendental degree $m$ over $S=\operatorname{Spec} k$, then $\operatorname{dim}_{S} X=m$. Note that this definition of dimension is different from that of relative dimensions. For instance, if $S=\operatorname{Spec} D$ and $X$ has relative dimension $d$ over $S$, then $\operatorname{dim}_{S} X=d+1$. We use $X_{(i)}$ (resp. $X^{(i)}$ ) to denote the set of dimension $i$ (resp. codimension $i$ ) points of $X$. For $x \in X$, we use $i_{x}$ to denote the embedding of $x$ and $i_{x *}$ the push-forward of (étale) sheaves. The number $n$ is always less than $d=\operatorname{dim} X$.

\section{ACKNOWLEDGEMENTS}

I am grateful to my advisor Thomas Geisser for his instruction and help. I am indebted to the referee for pointing out a mistake in a previous version. I also want to thank Enxin Wu for helpful conversations.

\section{COMPARISON BETWEen Bloch's COMPleX AND Moser's COMPleX}

Bloch's cycle complex. Let

$$
\Delta^{i}=\Delta_{S}^{i}=S \times_{\mathbb{Z}} \operatorname{Spec} \mathbb{Z}\left[t_{0}, \ldots, t_{i}\right] /\left(\sum t_{j}-1\right) .
$$

We define $z_{n}(X, i)$ as the free abelian group generated by closed integral subschemes $Z \subset X \times \Delta^{i}$ that intersect all the faces properly and $\operatorname{dim}_{S} Z=n+i$. Then $z_{n}(-, i)$ is a 
sheaf in the Zariski and étale topology on $X$. The following complex is defined by Bloch ([2], or see [9] for the notations):

$$
\rightarrow z_{n}(-, i) \stackrel{d}{\rightarrow} \ldots \rightarrow z_{n}(-, 1) \rightarrow z_{n}(-, 0) \rightarrow 0,
$$

where

$$
d(Z)=\sum_{j}(-1)^{j}\left[Z \cap V\left(t_{j}\right)\right],
$$

with $V\left(t_{j}\right)$ the closed integral subscheme generated by $t_{j}$ and $\left[Z \cap V\left(t_{j}\right)\right]$ the linear combination of irreducible components of $Z \cap V\left(t_{j}\right)$ with coefficients intersection multiplicities. We define the complex $\mathbb{Z}_{X}^{c}(n)^{t}$ to be the complex of sheaves in the topology $t$ with $t=\mathrm{Zar}$ or ét, and put $z_{n}(-,-i-2 n)$ in (cohomological) degree $i$, i.e.,

$$
\left(\mathbb{Z}_{X}^{c}(n)^{t}\right)^{i}=z_{n}(-,-i-2 n) .
$$

In this paper we are mostly interested in étale sheaves, so by $\mathbb{Z}_{X}^{c}(n)$ we mean the complex of étale sheaves, unless otherwise stated. Note that $\mathbb{Z}_{X}^{c}(n)^{Z a r}$ satisfies Zariski descent. Since $\mathbb{Z}_{X}^{c}(n)$ is a complex of flat sheaves, the derived tensor product agrees with the usual tensor product of complexes. For an abelian group $A$, define

$$
A_{X}^{c}(n)=A \otimes \mathbb{Z}_{X}^{c}(n) .
$$

The complex $\mathbb{Z}_{X}^{c}(n)(X)$ is covariant for proper maps and contravariant for quasi-finite, flat maps. Let $\mathbb{Z}_{X}^{c}=\mathbb{Z}_{X}^{c}(0)$, and omit $X$ if there is no ambiguity. Define motivic Borel-Moore homology to be

$$
H_{i}^{\mathrm{BM}}(X / S, \mathbb{Z}(n)) \stackrel{\text { def }}{=} H_{i}\left(\mathbb{Z}_{X}^{c}(n)(X)\right)=H^{-i}\left(\mathbb{Z}_{X}^{c}(n)(X)\right) .
$$

This definition of $\mathbb{Z}_{X}^{c}(n)$ and $H_{i}^{\mathrm{BM}}(X / S, \mathbb{Z}(n))$ depend on the base scheme $S$. Unless otherwise specified, they will be defined over base $S$ and we will just write $\mathbb{Z}_{X}^{c}(n)$ and $H_{i}^{\mathrm{BM}}(X, \mathbb{Z}(n))$, respectively. As an example, if $F$ has transcendental degree $d$ over the base $k$, then

$$
H_{2 d+i}^{\mathrm{BM}}(F / k, \mathbb{Z}(d+n))=H_{i}^{\mathrm{BM}}(F / F, \mathbb{Z}(n)) .
$$

For a scheme $X$ of pure dimension $d$ over $S$,

$$
H_{i}^{\mathrm{BM}}(X, \mathbb{Z}(n))=C H^{d-n}(X, i-2 n) .
$$

For a scheme $X$ smooth over over a perfect field $k$ and $\operatorname{dim} X=d$,

$$
\mathbb{Z}_{X}^{c}(d-n)^{Z a r} \cong \mathbb{Z}(n)[2 d] .
$$

Here $\mathbb{Z}(n)$ is the motivic complex defined by Voevodsky, and the $\cong$ here is a quasiisomorphism. In particular, for a field $F$ of transcendental degree $d$ over $S=\operatorname{Spec} k$,

$$
H_{i}^{\mathrm{BM}}(F / k, \mathbb{Z}(n))=C H^{d-n}(F, i-2 n) \cong H^{2 d-i}(F, \mathbb{Z}(d-n)) .
$$

The following three theorem state important property of cycle complex, and we will use them in Section 4. The first one is the localization property of cycle complex.

Theorem 2.1 (Levine, [22, Theorem 0.7]). For any $X$, let $U \stackrel{j}{\longrightarrow} X$ be an open subscheme with closed complement $Y \stackrel{i}{\longrightarrow} X$. Then there is a distinguished triangle of complexes of Zariski sheaves

$$
\longrightarrow i_{*} \mathbb{Z}_{Y}^{c}(n)^{Z a r} \longrightarrow \mathbb{Z}_{X}^{c}(n)^{Z a r} \longrightarrow j_{*} \mathbb{Z}_{U}^{c}(n)^{Z a r} \longrightarrow .
$$

We will need a weak form of the Gersten Conjecture of higher Chow groups. 
Conjecture 2.2 (Conjecture $\mathcal{B}(n)$ ). Let $R$ be a regular local ring and $K$ be its function field. Then the canonical map

$$
H_{s}^{\mathrm{BM}}(R, \mathbb{Z} / m(n)) \rightarrow H_{s}^{\mathrm{BM}}(K, \mathbb{Z} / m(n))
$$

is injective for any $s$.

Lemma 2.3. If $X$ is regular with dimension $d$, and the conjecture $\mathcal{B}(n)$ is true for all the local rings of points $x \in X$, then the complexes $\mathbb{Z}_{X}^{c} / m(n)$ and $\mathbb{Z}_{X}^{c} / m(n)^{Z a r}$ are acyclic at degree $>-d-n$.

Proof. From the conjecture, we see that for any $x \in X$ with local ring $\mathcal{O}_{X, x}$ and function field $K_{x}$, the maps are injective

$$
H_{s}^{\mathrm{BM}}\left(\mathcal{O}_{X, x}, \mathbb{Z} / m(n)\right) \rightarrow H_{s}^{\mathrm{BM}}\left(K_{x}, \mathbb{Z} / m(n)\right) .
$$

But

$$
H_{s}^{\mathrm{BM}}\left(K_{x}, \mathbb{Z} / m(n)\right)=H^{2 d-s}\left(K_{x}, \mathbb{Z} / m(d-n)\right) .
$$

Here $H^{2 d-s}\left(K_{x}, \mathbb{Z} / m(d-n)\right.$ is Voevodsky's motivic cohomology, which vanishes for $s<d+n$ [36, Theorem 3.6]. Hence, $H_{s}^{\mathrm{BM}}\left(\mathcal{O}_{X, x}, \mathbb{Z}^{c} / m(n)\right)=0$ for $s<d+n$. It implies that $\mathcal{H}^{i}\left(\mathbb{Z}_{X}^{c} / m(n)^{Z a r}\right)=0$ for $i>-d-n$. The same property holds for $\mathbb{Z}_{X}^{c} / m(n)$. Hence we finish the proof.

Let $\epsilon$ be the map from étale site to Zariski site. The Beilinson-Lichtenbaum Conjecture claims that the following map

$$
\eta_{X}: \mathbb{Z}_{X}^{c} / m(n)^{Z a r} \rightarrow \tau_{\leq-d-n} R \epsilon_{*} \mathbb{Z}_{X}^{c} / m(n)
$$

is an quasi-isomorphism. Recall that the Bloch-Kato Conjecture claims that for a field $F$ and $m \in \mathbb{Z}$, the Galois symbol

$$
K_{n}^{M}(F) / m \rightarrow H^{n}\left(F_{\text {ét }}, \mu_{m}^{\otimes n}\right)
$$

is an isomorphism. Since it was proved by Voevodsky and Rost recently, we will refer it as the Rost-Voevodsky Theorem. It implies the Beilinson-Lichtenbaum Conjecture for smooth varieties over a field (see [35] if the field has characteristic 0, and [13] for positive characteristic case). We generalize it to general schemes over $S$, assuming the conjecture $\mathcal{B}(n)$. First we prove a lemma from homological algebra.

Lemma 2.4. Let $F: \mathcal{A} \rightarrow \mathcal{B}$ be a left exact functor, and assume that there are enough injectives in $\mathcal{A}$. Assume that $C^{*}$ is a (cochain) complex. Then

$$
\tau_{\leq n} R F\left(\tau_{\leq n} C^{*}\right) \cong \tau_{\leq n} R F C^{*} .
$$

Proof. Let $C^{*} \rightarrow I^{*}$ be an injective resolution, i.e., $I^{*}$ is a complex of injectives and is quasi-isomorphic to $C^{*}$. Let $A$ be the kernel of

$$
d_{n}: I^{n} \rightarrow I^{n+1} \text {. }
$$

Then we obtain an injective resolution of $A$ as follows:

$$
0 \longrightarrow A \hookrightarrow I^{n} \stackrel{d_{n}}{\longrightarrow} I^{n+1} \stackrel{c_{n+1}}{\longrightarrow} J^{n+1} \longrightarrow .
$$

Then the new complex

$$
\longrightarrow I^{n-1} \stackrel{d_{n-1}}{\longrightarrow} I^{n} \stackrel{d_{n}}{\longrightarrow} I^{n+1} \stackrel{c_{n+1}}{\longrightarrow} J^{n+1} \longrightarrow
$$

is an injective resolution of $\tau_{\leq n} C^{*}$, which we denote as $J^{*}$. Then for $m \leq n$,

$$
H^{m}\left(F I^{*}\right) \cong H^{m}\left(F J^{*}\right) \text {. }
$$


Hence we get the conclusion.

Theorem 2.5. Let $X$ be a pure dimensional scheme over $S$ with $S=\operatorname{Spec} k$ or $\operatorname{Spec} D$, and $\operatorname{dim}_{S} X=d$. Then there is an quasi-isomorphism

$$
\eta_{X}: \tau_{\leq-d-n} \mathbb{Z}_{X}^{c} / m(n)^{Z a r} \rightarrow \tau_{\leq-d-n} R \epsilon_{*} \mathbb{Z}_{X}^{c} / m(n) .
$$

If $X$ is regular and the conjecture $\mathcal{B}(n)$ with $\mathbb{Z} / m$-coefficients is true for all the points $x \in X$, then the truncation in front of the first item can be removed.

Proof. If $X$ is smooth over $S=\operatorname{Spec} k$, then the conclusion

$$
\mathbb{Z}_{X}^{c} / m(n)^{Z a r} \rightarrow \tau_{\leq-d-n} R \epsilon_{*} \mathbb{Z}_{X}^{c} / m(n)
$$

is implied by the Rost-Voevodsky Theorem. If $X$ is a general variety over $\operatorname{Spec} k$ we prove the conjecture by induction on the dimension of $X$. Suppose that it is true for $Z$ such that $\operatorname{dim} Z<d$. Now suppose $\operatorname{dim} X=d$. We can assume that $X$ is reduced. Let $m=-d-n$ and $U \stackrel{j}{\longrightarrow} X$ be an smooth open subscheme with $Z \stackrel{i}{\longrightarrow} X$ its complement. Enlarge $Z$ so that it has pure codimension 1. From Theorem 2.1, there is a distinguished triangle

$$
\longrightarrow i_{*} \mathbb{Z}_{Z}^{c} / m(n)^{Z a r} \longrightarrow \mathbb{Z}_{X}^{c} / m(n)^{Z a r} \longrightarrow j_{*} \mathbb{Z}_{U}^{c} / m(n)^{Z a r} \longrightarrow .
$$

Apply the functor $\epsilon^{*}$, then apply the functor $R \epsilon_{*}$, we obtain a distinguished triangle

$$
R \epsilon_{*}\left(\epsilon^{*} i_{*} \mathbb{Z}_{Z}^{c} / m(n)^{Z a r}\right) \longrightarrow R \epsilon_{*}\left(\epsilon^{*} \mathbb{Z}_{X}^{c} / m(n)^{Z a r}\right) \longrightarrow R \epsilon_{*}\left(\epsilon^{*} j_{*} \mathbb{Z}_{U}^{c} / m(n)^{Z a r}\right) .
$$

Note that [10, Proposition 2.2]

$$
\epsilon^{*} \mathbb{Z}_{X}^{c} / m(n)^{Z a r}=\mathbb{Z}_{X}^{c} / m(n), \quad R \epsilon_{*}\left(\epsilon^{*} i_{*} \mathbb{Z}_{Z}^{c} / m(n)^{Z a r}\right)=i_{*} R \epsilon_{*}\left(\mathbb{Z}_{Z}^{c} / m(n)\right) .
$$

By induction assumption,

$$
\tau_{\leq-(d-1)-n} i_{*} R \epsilon_{*}\left(\mathbb{Z}_{Z}^{c} / m(n)\right) \cong \tau_{\leq-(d-1)-n} i_{*} \mathbb{Z}_{Z}^{c} / m(n)^{Z a r} .
$$

Hence, by the five lemma, it suffices to show that

$$
\tau_{\leq m} R \epsilon_{*}\left(\epsilon^{*} j_{*} \mathbb{Z}_{U}^{c} / m(n)^{Z a r}\right) \cong \tau_{\leq m} j_{*} \mathbb{Z}_{U}^{c} / m(n)^{Z a r} .
$$

That is, we only have to compare $R^{i} \epsilon_{*}\left(\epsilon^{*} j_{*} \mathbb{Z}_{U}^{c} / m(n)^{Z a r}\right)$ with $H^{i}\left(j_{*} \mathbb{Z}_{U}^{c} / m(n)^{Z a r}\right)$ for $i \leq m$. The former one is the Zariski sheaf associated to the presheaf sending $V$ to

$$
H_{e t}^{i}\left(V, \epsilon^{*} j_{*} \mathbb{Z}_{U}^{c} / m(n)^{Z a r}\right),
$$

while the latter one is the Zariski sheaf associated to the presheaf sending $V$ to (note that $\mathbb{Z}^{c} / m(n)^{Z a r}$ satisfies Zariski descent)

$$
H_{Z a r}^{i}\left(V \times{ }_{X} U, \mathbb{Z}_{U}^{c} / m(n)^{Z a r}\right) .
$$

Since $U$ is smooth, $\mathbb{Z}_{U}^{c} / m(n)^{Z a r}$ satisfies the Beilinson-Lichtenbaum Conjecture, so the two sheaves are isomorphic. Hence,

$$
\tau_{\leq-d-n} \mathbb{Z}_{X}^{c} / m(n)^{Z a r} \rightarrow \tau_{\leq-d-n} R \epsilon_{*} \mathbb{Z}_{X}^{c} / m(n) .
$$

If $X$ is a smooth scheme over $S=\operatorname{Spec} D$ with perfect residue fields, then the isomorphism

$$
\mathbb{Z}_{X}^{c} / m(n)^{Z a r} \rightarrow \tau_{\leq-d-n} R \epsilon_{*} \mathbb{Z}_{X}^{c} / m(n)
$$

is proved in [10, Theorem 1.2(2)]. Now let $X$ be a scheme flat over $D$. Since this is a local problem, we can assume that $D$ is a discrete valuation ring. Let $U$ be the generic fiber and $Z$ be the special fiber. Then $\operatorname{dim}_{S} Z=d-1$. So we have a localization diagram similar as 
above. Both $U$ and $Z$ are varieties over fields, and $U$ is smooth, so similarly, we can prove the first part.

If the conjecture $\mathcal{B}(n)$ with $\mathbb{Z} / m$-coefficients is true, from Lemma 2.3 , we get the conclusion.

For the reader's convenience, we recall Levine's proof of a weak form of the Kummer isomorphism for regular schemes over Dedekind domains.

Theorem 2.6 (Levine, [23, Theorem 12.5]). If $X$ is a regular and pure-dimension d scheme over $S=\operatorname{Spec} D$ or Spec $k, m$ is invertible in $S$. Then the following map is an isomorphism

$$
\tau_{\leq-d-n} \mathbb{Z}_{X}^{c} / m(n) \cong \mu_{m}^{\otimes d-n}[2 d] .
$$

If the conjecture $\mathcal{B}(n)$ with $\mathbb{Z} / m$-coefficients holds for all the points $x \in X$, then the truncation can be removed.

Proof. If $X$ is smooth over a field, then it is proved in [13, Theorem 1.5]. If $X$ is smooth over $D$, then it is proved in [10, Theorem 1.2(4)] (in this case the map $\varrho_{X}: \mathbb{Z}_{X}^{c} / m(n) \rightarrow$ $\mu_{m}^{\otimes d-n}[2 d]$ is defined in $\left.[23, \S 12]\right)$. The only case left is when $X$ is a regular scheme flat over $D$, so $d \geq 1$. This is a local problem, so assume that $D$ is a discrete valuation ring with perfect residue field of characteristic coprime to $m$.

First we claim that, if $X$ is regular and admits an closed-open decomposition

$$
X=Z \coprod U
$$

such that $Z$ smooth and the conclusion holds for $U$, then it is also true for $X$.

By localization sequence of cycle complex and $\mu_{m}$, there is a commutative diagram

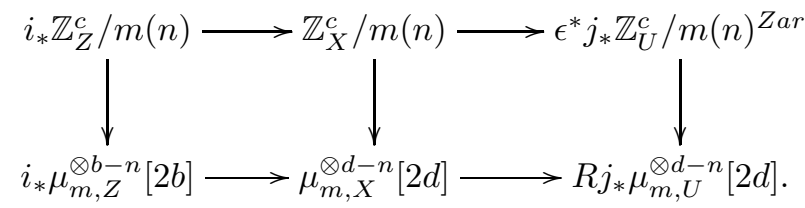

Here the two rows are distinguished triangles, and $b=\operatorname{dim} Z$. The existence of the lower distinguished triangle is due to Gabber, [8]. Then

$$
R^{s}\left(j_{*} \mu_{m, U}^{\otimes d-n}[2 d]\right)=\left\{\begin{array}{cc}
\mu_{m, X}^{\otimes d-n}[2 d], & s=-2 d, \\
i_{*} \mu_{m, Z}^{b-n}[2 b], & s=-1-2 b, \\
0, & \text { otherwise. }
\end{array}\right.
$$

Moreover, we have

$$
\tau_{\leq-d-n} \epsilon^{*} j_{*} \mathbb{Z}_{U}^{c} / m(n)^{Z a r} \cong \tau_{\leq-d-n} R j_{*} \mathbb{Z}_{U}^{c} / m(n) \cong \tau_{\leq-d-n}\left(R j_{*} \mu_{m, U}^{\otimes d-n}[2 d]\right) .
$$

Here the first isomorphism follows from the Beilinson-Lichtenbaum Conjecture for regular $U$ (Theorem 2.5), and the second one follows from the assumption on $U$.

If $n \leq b$, then since $Z$ is smooth, we have isomorphism

$$
\mathbb{Z}_{Z}^{c} / m(n) \cong \mu_{m, Z}^{\otimes b-n}[2 b] .
$$

Hence, the two distinguished triangles have isomorphic cohomology up to degree $-d-n$. In particular,

$$
\tau_{\leq-d-n} \mathbb{Z}_{X}^{c} / m(n) \cong \tau_{\leq-d-n}\left(\mu_{m, X}^{\otimes d-n}[2 d]\right) \cong \mu_{m, X}^{\otimes d-n}[2 d] .
$$


If $n>b$, then $\mathbb{Z}_{Z}^{c} / m(n)=0$. Then

$$
\tau_{\leq-d-n} \mathbb{Z}_{X}^{c} / m(n) \cong \tau_{\leq-d-n} \epsilon^{*} j_{*} \mathbb{Z}_{U}^{c} / m(n)^{Z a r} \cong \tau_{\leq-d-n}\left(R j_{*} \mu_{m, U}^{\otimes d-n}[2 d]\right) .
$$

But $b \leq d-1$ and $n>b$ imply that $-2 d \leq-d-n<-1-2 b$. So

$$
\tau_{\leq-d-n}\left(R j_{*} \mu_{m, U}^{\otimes d-n}[2 d]\right) \cong R^{-2 d}\left(j_{*} \mu_{m, U}^{\otimes d-n}[2 d]\right) \cong \mu_{m, X}^{\otimes d-n}[2 d] .
$$

This finishes the proof of the claim.

Now for $X$ regular over $D$, we can find $U$ and a filtration of $X$

$$
\emptyset=Z_{t+1} \subset Z_{t} \subset \ldots \subset Z_{1} \subset Z_{0}=X
$$

such that $Z_{1}$ and $U$ are the special and generic fiber of $X$, respectively, and $Z_{i+1}$ is the singular locus of $Z_{i}$. Then we have the following decompositions

$$
\begin{gathered}
X-Z_{2}=\left(Z_{1}-Z_{2}\right) \coprod\left(X-Z_{1}\right), \\
X-Z_{3}=\left(Z_{2}-Z_{3}\right) \coprod\left(X-Z_{2}\right), \\
\cdots \\
X=X-Z_{t+1}=\left(Z_{t}-Z_{t+1}\right) \coprod\left(X-Z_{t}\right)
\end{gathered}
$$

Here all the $Z_{i}-Z_{i+1}$ and $X-Z_{1}=U$ are smooth, and all the $X-Z_{i}$ are regular. From the first decomposition, we get the existence of the isomorphism for $X-Z_{2}$. Inductively, we get that the conclusion is true for $X=X-Z_{t+1}$.

If the conjecture $\mathcal{B}(n)$ with $\mathbb{Z} / m$-coefficients is true, then the truncation on $\mathbb{Z}_{X}^{c} / m(n)$ is not necessary.

The following are the duality results by Geisser, [9].

Theorem 2.7 (Geisser, [9, Theorem 4.1]). If $f: X \rightarrow$ Spec $k$ is separated and of finite type over a perfect field, then for any constructible sheaf $\mathcal{F}$ on $X$, there is a canonical quasi-isomorphism

$$
\operatorname{RHom}_{X}\left(\mathcal{F}, \mathbb{Z}_{X}^{c}\right) \cong \mathrm{RHom}_{\text {Spec } k}\left(R f_{!} \mathcal{F}, \mathbb{Z}\right) .
$$

Corollary 2.8 (Geisser, [9, §5]). (1) Let $S$ be the spectrum of a number ring or a finite field, and $\mathcal{F}$ be a constructible sheaf on $X$, then there are perfect pairings of finite groups

$$
H_{c}^{i}\left(X_{e ́ t}, \mathcal{F}\right) \times \operatorname{Ext}_{X}^{2-i}\left(\mathcal{F}, \mathbb{Z}_{X}^{c}\right) \rightarrow \mathbb{Q} / \mathbb{Z}
$$

(2)Let $k$ be an algebraically closed field and $\mathcal{F}$ be a constructible sheaf on $X$. Then there are perfect pairings of finitely generated groups

$$
H_{c}^{i}\left(X_{e ́ t}, \mathcal{F}\right) \times \operatorname{Ext}_{X}^{1-i}\left(\mathcal{F}, \mathbb{Z}_{X}^{c}\right) \rightarrow \mathbb{Q} / \mathbb{Z}
$$

Niveau Spectral Sequence. The method of this section depends highly on the niveau spectral sequence of higher Chow groups, so let us recall its construction. We adopt the notation in [9. Proposition 2.1]. We only define it for $\mathbb{Z}$-coefficients. Such spectral sequence with other coefficients is totally analogous. Let $p$ be the projection $X \times \Delta^{i} \rightarrow X$. Let $F_{s}=F_{s} \mathbb{Z}^{c}(n)(X)$ be the subcomplex generated by cycles $Z \subset X \times \Delta^{i}$ with $\operatorname{dim} p(Z) \leq s$, so

$$
\left(F_{s}\right)_{m}=\left(F_{s}\right)^{-m} \subset\left(\mathbb{Z}^{c}(n)(X)\right)^{-m}=z_{n}(X, m-2 n) .
$$

There is a short exact sequence of complexes:

$$
0 \rightarrow F_{s-1} \rightarrow F_{s} \rightarrow F_{s} / F_{s-1} \rightarrow 0
$$


which induces a long exact sequence of abelian groups

$$
\rightarrow H_{s+t+1}\left(F_{s} / F_{s-1}\right) \rightarrow H_{s+t}\left(F_{s-1}\right) \rightarrow H_{s+t}\left(F_{s}\right) \rightarrow H_{s+t}\left(F_{s} / F_{s-1}\right) \rightarrow .
$$

Moreover, by the localization property of higher Chow groups, we have

$$
H_{s+t}\left(F_{s} / F_{s-1}\right) \cong \bigoplus_{X_{(s)}} H_{s+t}^{\mathrm{BM}}(k(x), \mathbb{Z}(n)) .
$$

Therefore, there is a convergent spectral sequence:

$$
E_{s, t}^{1}=\bigoplus_{X_{(s)}} H_{s+t}^{\mathrm{BM}}(k(x), \mathbb{Z}(n)) \Rightarrow H_{s+t}^{\mathrm{BM}}(X, \mathbb{Z}(n)) .
$$

This construction induces a filtration

$$
N_{s} H_{m}^{\mathrm{BM}}(X, \mathbb{Z}(n)) \stackrel{\text { def }}{=} \operatorname{Im}\left(H_{m}\left(F_{s}\right) \rightarrow H_{m}^{\mathrm{BM}}(X, \mathbb{Z}(n))\right),
$$

which is called the niveau filtration of higher Chow groups. Note that $E_{s, t}^{1}=0$ for $t<n$.

From the niveau spectral sequence (2.0.4), we can define $C_{X}^{\mathrm{HC}}(n) \stackrel{\text { def }}{=} E_{*, n}^{1}$ with

$$
\left(C_{X}^{\mathrm{HC}}(n)\right)^{i}=\bigoplus_{X_{(-i-n)}} H_{-i}^{\mathrm{BM}}(k(x), \mathbb{Z}(n))=\bigoplus_{X_{(-i-n)}} C H^{-i-2 n}(k(x),-i-2 n) .
$$

and $C_{X}^{\mathrm{HC}}(n) / p^{r}$ to be the corresponding complex with terms

$$
\bigoplus_{X_{(-i-n)}} H_{-i}^{\mathrm{BM}}\left(k(x), \mathbb{Z} / p^{r}(n)\right) .
$$

If $X$ is a scheme over a perfect field of characteristic $p>0$, then

$$
H_{-i}^{\mathrm{BM}}\left(k(x), \mathbb{Z} / p^{r}(n)\right) \cong H_{-i}^{\mathrm{BM}}(k(x), \mathbb{Z}(n)) / p^{r},
$$

and $C_{X}^{\mathrm{HC}}(n) / p^{r}$ is exactly the complex $C_{X}^{\mathrm{HC}}(n)$ modulo $p^{r}$, so in this case our notation causes no ambiguity. Denote by $\left(C_{X}^{\mathrm{HC}}(n)\right)^{t}$ the sheafification in topology $t$ of the complex of presheaves $C_{X}^{\mathrm{HC}}(n)$. This complex $\left(C_{X}^{\mathrm{HC}}(n)\right)^{t}$ is called the Gersten complex of sheaf $H_{d+n}\left(\mathbb{Z}_{X}^{c}(n)^{t}\right)$. It is called the Gersten resolution of $H_{d+n}\left(\mathbb{Z}_{X}^{c}(n)^{t}\right)$ if they are quasiisomorphic via the canonical map

$$
H_{d+n}\left(\mathbb{Z}_{X}^{c}(n)^{t}\right) \rightarrow \bigoplus_{X_{(d)}} a_{t} i_{x, p} H_{d+n}^{\mathrm{BM}}(-, \mathbb{Z}(n)) .
$$

Here $a_{t}$ is denoted as the sheafification in topology $t$, and $i_{x, p}$ is the direct image of presheaves along the embedding $i_{x}: x \rightarrow X$. Note that $a_{t} i_{x, p} \neq i_{x *} a_{t}$ if $t=e ́ t$ and $i_{x *}$ is the direct image of étale sheaves.

There is a map of complexes

$$
\phi: \mathbb{Z}_{X}^{c}(n)(X) \rightarrow C_{X}^{\mathrm{HC}}(n)
$$

which induces the edge morphisms of the spectral sequence. More precisely,

$$
\phi_{m}:\left(\mathbb{Z}_{X}^{c}(n)(X)\right)_{m}=z_{n}(X, m-2 n) \rightarrow \bigoplus_{X_{(m-n)}} H_{m}^{\mathrm{BM}}(k(x), \mathbb{Z}(n))=\left(C_{X}^{\mathrm{HC}}(n)\right)_{m}
$$

satisfies the following properties:

i) if $m>n+d$, then $m-n>d$ and $\phi_{m}=0$; similarly, if $m<n, \phi_{m}=0$ as well.

ii) if $n \leq m \leq n+d$, then $\phi_{m}$ is the composition:

$z_{n}(X, m-2 n)=\left(F_{m-n}\right)_{m} \rightarrow \frac{\left(F_{m-n}\right)_{m}}{\left(F_{m-n-1}\right)_{m}} \cong \bigoplus_{X_{(m-n)}} z_{n}(k(x), m-2 n) \rightarrow \bigoplus_{X_{(m-n)}} H_{m}^{\mathrm{BM}}(k(x), \mathbb{Z}(n))$. 
Gersten complex of logarithmic de Rham-Witt sheaves. Let $X$ be a scheme essentially of finite type over $k$ of characteristic $p>0$. If $m \geq 0$, then define the logarithmic de Rham-Witt sheaf $W_{r} \Omega_{X, \log }^{m}$ to be the étale subsheaf of $W_{r} \Omega_{X}^{m}$ generated by elements of the form

$$
d \log f_{1} \wedge \ldots \wedge d \log f_{m}
$$

with $f_{i} \in \mathcal{O}_{X}^{*}$. Define $W_{r} \Omega_{X, \log }^{m}=0$ if $m<0$. If $X=\operatorname{Spec} F$ is a field, we use the notation $\nu_{F, r}^{m}=W_{r} \Omega_{X, \log }^{m}$. If $X$ is smooth, define $\widetilde{\nu}_{X, r}(n)$ to be the Gersten complex of logarithmic de Rham-Witt sheaves:

$$
0 \rightarrow \bigoplus_{X_{(d)}} i_{x *} \nu_{k(x), r}^{d-n} \rightarrow \ldots \rightarrow \bigoplus_{X_{(1)}} i_{x *} \nu_{k(x), r}^{1-n} \rightarrow \bigoplus_{X_{(0)}} i_{x *} \nu_{k(x), r}^{-n} \rightarrow 0 .
$$

The term indexed by $X_{(-i-n)}$ is in (cohomological) degree $i$, i.e.,

$$
\widetilde{\nu}_{X, r}(n)^{i}=\bigoplus_{X_{(-i-n)}} \nu_{k(x), r}^{-i-2 n} .
$$

Define $\widetilde{\nu}_{X, r}=\widetilde{\nu}_{X, r}(0)$. The differentials of $\widetilde{\nu}_{X, r}(n)$ are induced from the coniveau filtration of the sheaf $W_{r} \Omega_{X, \log }^{d-n}$, similar as the one for higher Chow groups above. If $X$ is not smooth, using Theorem 2.10 below, Moser [27] also defines $\widetilde{\nu}_{X, r}(n)(X)$ by identifying it with $C_{X}^{\mathrm{M}}(n) / p^{r}$ (see definition below). The following is the duality result of Moser.

Theorem 2.9 (Moser, [27, Theorem 5.6]). Let $k$ be a finite field of characteristic $p$ and $X$ be a $k$-scheme of pure dimension $d$. Then for every $r \geq 1$ and every constructible $\mathbb{Z} / p^{r}$-sheaf $\mathcal{F}$, there are perfect pairings of finite groups

$$
H_{c}^{i}\left(X_{\tilde{e} t}, \mathcal{F}\right) \times \operatorname{Ext}_{X}^{1-i}\left(\mathcal{F}, \widetilde{\nu}_{X, r}\right) \rightarrow \mathbb{Z} / p^{r}
$$

Kato's complex of Milnor K-theory. Let $X$ be a scheme over $S$. Define $C_{X}^{\mathrm{M}}(n)$ to be Kato's complex of Milnor K-theory (cf. K. Kato, [19]):

$$
0 \rightarrow \bigoplus_{X_{(d)}} K_{d-n}^{M}(k(x)) \stackrel{d^{\prime}}{\rightarrow} \ldots \rightarrow \bigoplus_{X_{(1)}} K_{1-n}^{M}(k(x)) \rightarrow \bigoplus_{X_{(0)}} K_{-n}^{M}(k(x)) \rightarrow 0 .
$$

The group $K_{m}^{M}(k(x))$ is Milnor K-group, and $K_{m}^{M}(k(x))=0$ if $m<0$. The differential $d^{\prime}$ is defined as follows: for any $x \in X_{(m)}$ and any $y \in \overline{\{x\}} \cap X_{(m-1)}$, we take the normalization $\overline{\left\{x^{\prime}\right\}}$ of $\overline{\{x\}}$ with $x^{\prime}$ its generic point and define a map

$\partial_{y}: K_{m-n}^{M}(k(x))=K_{m-n}^{M}\left(k\left(x^{\prime}\right)\right) \stackrel{\sum \partial_{y^{\prime}}}{\longrightarrow} \bigoplus_{y^{\prime} \mid y} K_{m-n-1}^{M}\left(k\left(y^{\prime}\right)\right) \stackrel{\sum N_{k\left(y^{\prime} / k(y)\right.}}{\longrightarrow} K_{m-n-1}^{M}(k(y))$.

Here the notation $y^{\prime} \mid y$ means that $y^{\prime} \in{\overline{\left\{x^{\prime}\right\}}}_{(m-1)}$ is in the fiber of $y$,

$$
N_{k\left(y^{\prime}\right) / k(y)}: K_{m-n-1}^{M}\left(k\left(y^{\prime}\right)\right) \rightarrow K_{m-n-1}^{M}(k(y))
$$

is the norm map of Milnor K-theory (see Bass-Tate, [1] and Kato, [18, Section 1.7]), and $\partial_{y^{\prime}}$ is the tame symbol defined by $y^{\prime}$. Then

$$
d^{\prime} \stackrel{\text { def }}{=} \sum_{y \in X_{(m-1)} \cap \overline{\{x\}}} \partial_{y} .
$$

Note that the sum in $d^{\prime}$ is finite since elements in $K_{m-n}^{M}\left(k\left(x^{\prime}\right)\right)$ are represented by sums of tensors of $m-n$ elements in $k\left(x^{\prime}\right)^{*}$, and each element in $k\left(x^{\prime}\right)^{*}$ has a finite number of 
poles and zeros. When applying the tame symbol, only a finite number of terms in the sum are non-zero. Suppose that in (cohomological) degree $i$,

$$
C_{X}^{\mathrm{M}}(n)^{i}=\bigoplus_{X_{(-i-n)}} K_{-i-2 n}^{M}(k(x)),
$$

and define $C_{X}^{\mathrm{M}}=C_{X}^{\mathrm{M}}(0)$. The complex $C_{X}^{\mathrm{M}}(n)$ is covariant for proper maps and contravariant for quasi-finite and flat maps (see Rost, [31, Proposition 4.6(1),(2)]).

Note that all the three complexes, $C_{X}^{\mathrm{HC}}(n), C_{X}^{\mathrm{M}}(n)$ and $\widetilde{\nu}_{X, r}(n)$ are concentrated in cohomological degree $[-n-d,-2 n]$ when $n \leq d=\operatorname{dim} X$.

Theorem 2.10 (Bloch-Kato, Gros-Suwa, Moser). For X a scheme separated and essentially of finite type over $S=\operatorname{Spec} k$ with $k$ perfect of characteristic $p>0$,

$$
C_{X}^{M}(n) / p^{r} \cong \widetilde{\nu}_{X, r}(n)(X) .
$$

Proof. By Bloch-Kato, [4, Theorem 2.1], for any field $F$, there is an isomorphism

$$
K_{n}^{M}(F) / p^{r} \cong \nu_{F, r}^{n}(F)
$$

sending $\left\{f_{1}, \ldots, f_{n}\right\}$ to

$$
d \log f_{1} \wedge \ldots \wedge d \log f_{n} .
$$

By [15, Lemma 4.1] (see Jannsen-Saito-Sato, [16, Theorem 2.1.1 and Theorem 2.11.3(3)] for a more detailed proof), this isomorphism respects the differentials in $C_{X}^{\mathrm{M}}(n)$ and $\widetilde{\nu}_{X, r}(n)(X)$. Hence it induces an isomorphism of complexes

$$
C_{X}^{\mathrm{M}}(n) / p^{r} \stackrel{\cong}{\longrightarrow} \widetilde{\nu}_{X, r}(n)(X) .
$$

To relate $C_{X}^{\mathrm{HC}}(n)$ with $C_{X}^{\mathrm{M}}(n)$, we need the Nesterenko-Suslin isomorphism.

Theorem 2.11 (Nesterenko-Suslin, [29], Theorem 4.9). Let $F$ be a field of transcendental degree $d$ over a field $k$, and $n \leq d$. Then there is an isomorphism

$$
\chi_{F}: H_{d+n}^{\mathrm{BM}}(F, \mathbb{Z}(n)) \rightarrow K_{d-n}^{M}(F) .
$$

Proof. The map $\chi_{F}$ is defined as follows: for any generator $z \in z_{n}(F, d-n), \chi_{F}(\bar{z})=$ $N\left(\beta_{z}\right)$. Here $\bar{z}$ is the image of $z$ in $H_{d+n}^{\mathrm{BM}}(F, \mathbb{Z}(n))$,

$$
N: K_{d-n}^{M}(k(z)) \rightarrow K_{d-n}^{M}(F)
$$

is the norm map of Milnor K-theory, and

$$
\beta_{z}=\left\{\frac{-t_{0}}{t_{d-n}}, \ldots, \frac{-t_{d-n-1}}{t_{d-n}}\right\} \in K_{d-n}^{M}(k(z))
$$

with $t_{i}$ 's the coordinates of $z$ in $\Delta_{F}^{d-n}$, i.e., pull-back of $t_{i}$ on $z$. Since $z$ intersects all the faces properly, $t_{i} \in k(z)^{*}$ for all $i$. Nestenrenko and Suslin showed that $\chi_{F}$ is an isomorphism.

It is easy to see that this isomorphism does not depend on the choice of the base field $k$. In particular, let $F=k$, and $n \leq 0$. Then $d=0$ and we have isomorphism

$$
\chi_{F}: H_{n}^{\mathrm{BM}}(F, \mathbb{Z}(n)) \cong K_{-n}^{M}(F) .
$$


Lemma 2.12. For $X$ separated and essentially of finite type over $S=\operatorname{Spec} k$ or $S=$ Spec $D$, the Nesterenko-Suslin isomorphism induces an isomorphism of complexes

$$
\chi: C_{X}^{H C}(n) \rightarrow C_{X}^{M}(n) .
$$

If the base is Spec $k$ with characteristic $p>0$, then

$$
\chi / p^{r}: C_{X}^{H C}(n) / p^{r} \stackrel{\cong}{\longrightarrow} C_{X}^{M}(n) / p^{r} .
$$

Proof. We have to show that the maps

$$
\chi:\left(C_{X}^{\mathrm{HC}}(n)\right)_{m} \rightarrow\left(C_{X}^{M}(n)\right)_{m}
$$

induces the following commutative diagram:

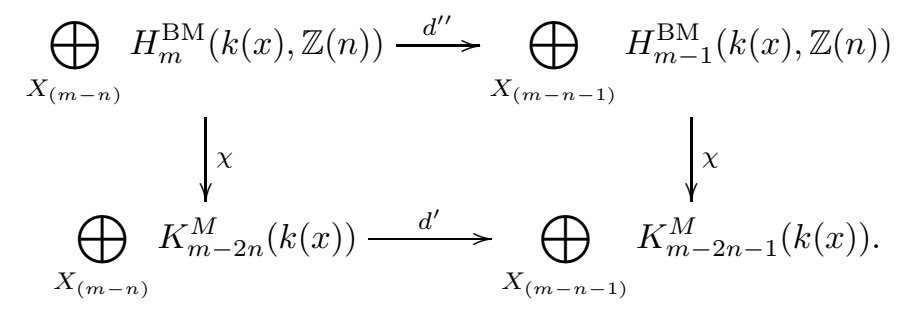

It suffices to prove the conclusion for $m=d+n$ with $d=\operatorname{dim} X$, since commutativity of such diagram at the other degrees is similar. Let $X^{\prime} \rightarrow X$ be the normalization of $X$, and $x$ be a codimension 1 point of $X$. Consider the following commutative diagram:

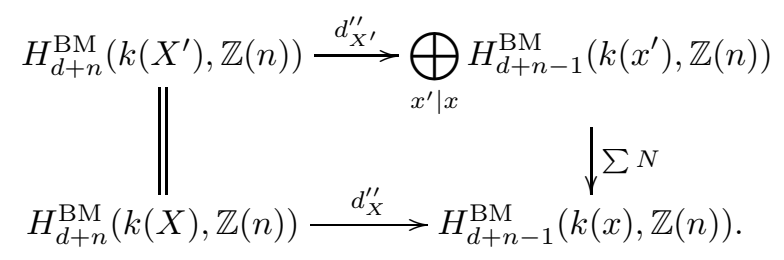

Here $d_{X}^{\prime \prime}$ and $d_{X^{\prime}}^{\prime \prime}$ are differentials in $C_{X}^{\mathrm{HC}}(n)$ and $C_{X^{\prime}}^{\mathrm{HC}}(n)$, respectively, and $N=N_{k\left(x^{\prime}\right) / k(x)}$ is the push-forward of higher Chow groups of finite field extensions. This diagram is commutative by covariance of Gersten complex. So

$$
d_{X}^{\prime \prime}=\sum N \circ d_{X^{\prime}}^{\prime \prime}
$$

To finish the proof, consider the following diagram:

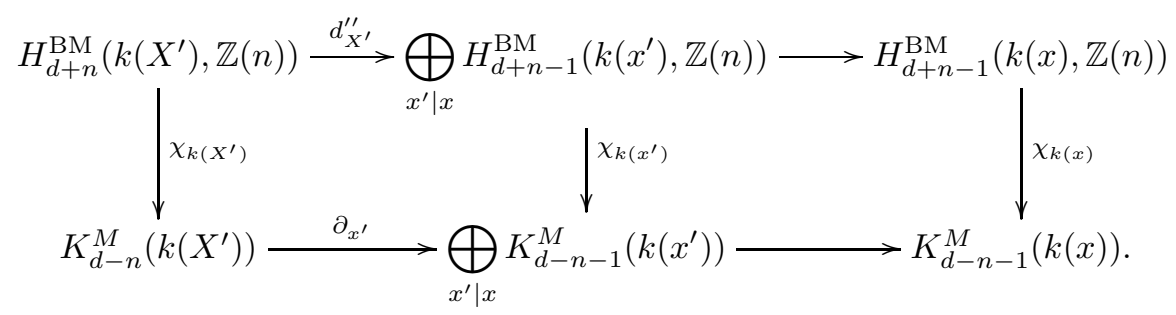

The horizontal maps on the right hand square are norm maps. By definition, the composition of the maps at the bottom is the differential $d^{\prime}$ in $C_{X}^{\mathrm{M}}(n)$. By the first part of the proof, the composition of the maps on the top is the differential $d^{\prime \prime}$ in $C_{X}^{\mathrm{HC}}(n)$. Hence it suffices to prove commutativity of this diagram. The right hand diagram commutes by [29, Lemma 4.7]. On the other hand, Geisser and Levine showed commutativity of the left hand square [14, Lemma 3.2] (even though their statement is for $\mathbb{Z} / p$-coefficients, their proof 
holds for $\mathbb{Z}$-coefficients as well). Hence we prove the first part. The second part about the coefficients $\mathbb{Z} / p^{r}$ holds since both complexes are $C_{X}^{M}(n)$ and $C_{X}^{\mathrm{HC}}(n)$ modular $p^{r}$.

Definition 2.13. Define

$$
\psi=\chi \circ \phi: \mathbb{Z}_{X}^{c}(n)(X) \rightarrow C_{X}^{H C}(n) \stackrel{\cong}{\rightarrow} C_{X}^{M}(n) .
$$

Explicitly,

Definition 2.14. Denote $p: X \times \Delta^{j} \rightarrow X$. Given a generator

$$
Z \in z_{n}(X, m-2 n)=\left(\mathbb{Z}_{X}^{c}(n)(X)\right)_{m},
$$

we define

$$
\psi_{m}(Z) \in \bigoplus_{X_{(m-n)}} K_{m-2 n}^{M}(k(x))=C_{X}^{M}(n)_{m}
$$

(see (2.0.5)) as follows:

1) if $m>n+d$ or $m<n, \psi_{m}(Z) \stackrel{\text { def }}{=} 0$.

2) if $n \leq m \leq n+d$ and $\operatorname{dim} p(Z)<m-n, \psi_{m}(Z) \stackrel{\text { def }}{=} 0$.

3) if $n \leq m \leq n+d$ and $\operatorname{dim} p(Z)=m-n$, then $Z$ is dominant over some $x \in$ $X_{(m-n)}$. Pulling back $Z$ along

$$
\operatorname{Spec} k(x) \rightarrow X,
$$

we obtain $Z_{x} \in z_{n}(k(x), m-2 n)$, which is sent to $\bar{Z}_{x}$ by the quotient

$$
z_{n}(k(x), m-2 n) \rightarrow H_{m}^{\mathrm{BM}}(k(x), \mathbb{Z}(n)) .
$$

Applying the Nesterenko-Suslin isomorphism $\chi_{k(x)}$, we get

$$
\psi_{m}(Z) \stackrel{\text { def }}{=} \chi_{k(x)}\left(\bar{Z}_{x}\right) \in K_{m-2 n}^{M}(k(x)) .
$$

Since $Z$ is dominant over $x, Z_{x}$ is a closed point in $\Delta_{k(x)}^{m-2 n}$ with residue field $k\left(Z_{x}\right)=$ $k(Z)$. Therefore, by definition of $\chi_{k(x)}$,

$$
\psi_{m}(Z)=N_{k(Z) / k(x)}\left(\beta_{Z}\right)
$$

with $t_{i} \in k(Z)^{*}$ and

$$
\beta_{Z}=\beta_{Z_{x}}=\left\{\frac{-t_{0}}{t_{m-2 n}}, \ldots, \frac{-t_{m-2 n-1}}{t_{m-2 n}}\right\} .
$$

Note that in Definition 2.14, case 3), $\bar{Z}_{x}=\phi_{m}(Z)$ where $\phi$ is the map from $\mathbb{Z}_{X}^{c}(n)(X)$ to $C_{X}^{H C}(n)$.

Theorem 2.15. Let $X$ be separated and essentially of finite type over Spec $k$ or $\operatorname{Spec} D$. The map $\psi$ defined above is a map of complexes, and it is functorial with respect to pullbacks defined by quasi-finite, flat maps and push-forwards defined by proper maps.

Proof. Since $\psi=\chi \circ \phi$, it is a map of complexes.

For functoriality, it suffices to assume that $n \leq m \leq n+d$. First, we show that $\psi$ is compatible with pull-backs defined by quasi-finite, flat maps $f: X \rightarrow Y$. We have to 
prove that the following diagram is commutative:

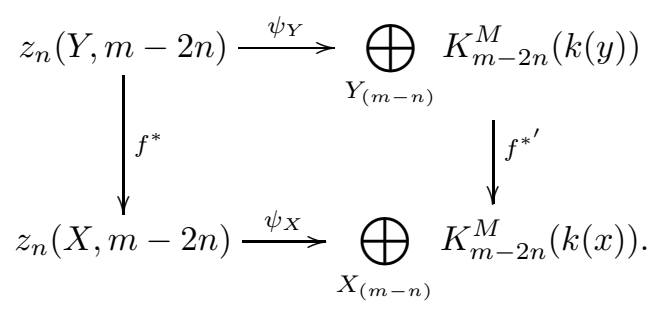

Here $f^{*}$ sends a generator $Z \in z_{n}(Y, m-2 n)$ to its cycle theoretic pull-back

$$
f^{-1}(Z) \in z_{n}(X, m-2 n),
$$

and

$$
f^{* \prime}: K_{m-2 n}^{M}(k(y)) \rightarrow K_{m-2 n}^{M}(k(x))
$$

is defined by the field extension $k(y) \subset k(x)$ with $x \in X_{(m-n)}, y \in Y_{(m-n)}$ and $f(x)=$ $y$. Let

$$
p_{X}: X \times \Delta^{m-2 n} \rightarrow X, p_{Y}: Y \times \Delta^{m-2 n} \rightarrow Y
$$

be the projections. If $\operatorname{dim} p_{Y}(Z)<m-n$, then $\operatorname{dim} p_{X}\left(f^{-1}(Z)\right)<m-n$, so

$$
\psi_{X} f^{*}(Z)=0=f^{*^{\prime}} \psi_{Y}(Z) .
$$

Suppose that $\operatorname{dim} p_{Y}(Z)=m-n$. Without loss of generality, replacing $Y$ by $p_{Y}(Z)$ and $X$ by $X \times_{Y} p(Z)$, we can assume that $Y$ is irreducible of dimension $m-n$ and $Z$ is dominant over $Y$. Since $f$ is quasi-finite and flat, $X$ is of equi-dimensional $m-n$. Let $X=\cup_{i} X_{i}, X_{i}$ be irreducible components of $X$ and $x_{i}$ be the generic points of $X_{i}$. Then $\operatorname{dim} X_{i}=m-n$. Therefore it suffices to prove commutativity of the following diagram:

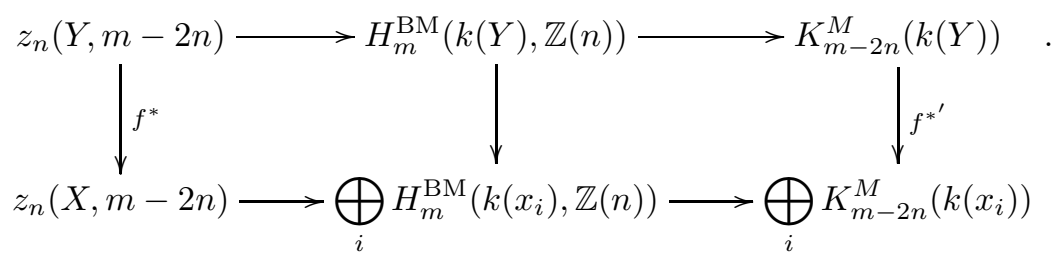

The square on the left commutes by functoriality of higher Chow groups with respect to flat pull back, the square on the right commutes since by definition, the Nesterenko-Suslin isomorphism is covariant with respect to finite field extensions. Therefore $\psi$ commutes with quasi-finite and flat pull-backs.

If $g: X \rightarrow Y$ is a proper map, then $\psi$ is covariant for push-forwards. To see that, fix

$$
Z \in z_{n}(X, m-2 n) .
$$

After replacing $X$ by the irreducible component of $X$ containing $p_{X}(Z)$, it suffices to assume that $X$ is irreducible of dimension $n$ with function field $K$ and prove that $Z$ is sent to the same element via the two paths in the following square:

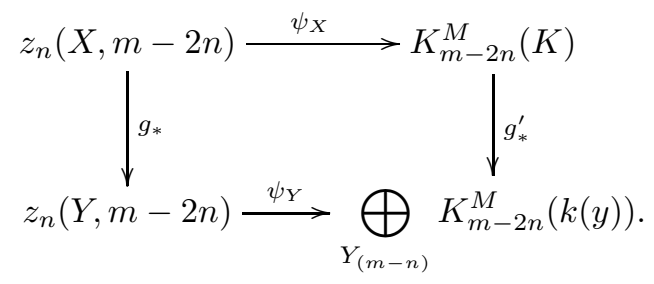


Here $g_{*}(Z)$ is defined as follows:

$$
g_{*}(Z)= \begin{cases}0, & \text { if } \operatorname{dim} g(Z)<m-n \\ m_{Z} \cdot g(Z), & \text { if } \operatorname{dim} g(Z)=m-n\end{cases}
$$

with $m_{Z}=[k(Z): k(g(Z))]$, and

$$
g_{*}^{\prime}= \begin{cases}0, & \text { if } \operatorname{dim} g(X)<m-n ; \\ N_{K / k(y)}, & \text { if } X \text { dominant over some } y \in Y_{(m-n)} .\end{cases}
$$

To show that $g_{*}^{\prime} \psi_{X}(Z)=\psi_{Y} g_{*}(Z)$, there are three cases:

$1)$ if $\operatorname{dim} g(X)<m-n$, then $g_{*}^{\prime}=0$. Moreover,

$$
\operatorname{dim} p_{Y}\left(g_{*}(Z)\right)=\operatorname{dim} g\left(p_{X}(Z)\right) \leq \operatorname{dim} g(X)<m-n .
$$

Hence $\psi_{Y}\left(g_{*}(Z)\right)=0$.

2) if $X$ is dominant over some $y \in Y_{(m-n)}$ and $\operatorname{dim} p_{X}(Z)<m-n$, then

$$
g_{*}^{\prime} \psi_{X}(Z)=g_{*}^{\prime}(0)=0
$$

Moreover, $\operatorname{dim} p_{X}(Z)<m-n$ also implies $\operatorname{dim} p_{Y}(g(Z))<m-n$, hence

$$
\psi_{Y} g_{*}(Z)=\psi_{Y}(0)=0 \text {. }
$$

3) if $X$ is dominant over some $y \in Y_{(m-n)}$ and $\operatorname{dim} p_{X}(Z)=m-n$, then

$$
\operatorname{dim} p_{Y}(g(Z))=\operatorname{dim} g\left(p_{X}(Z)\right)=m-n .
$$

Therefore, $Z$ is dominant over $X$ and $g(Z)$ is irreducible and dominant over $y$. We have a commutative diagram of field extensions:

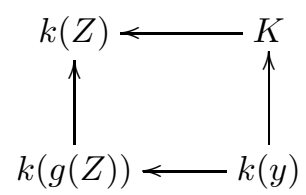

Then

and

$$
\psi_{Y} g_{*}(Z)=\psi_{Y}\left(m_{Z} \cdot g(Z)\right)=N_{k(g(Z)) / k(y)}\left(m_{Z} \cdot \beta_{g(Z)}\right)
$$

$$
g_{*}^{\prime} \psi_{X}(Z)=N_{K / k(y)} N_{k(Z) / K}\left(\beta_{Z}\right)=N_{k(g(Z)) / k(y)} N_{k(Z) / k(g(Z))}\left(\beta_{Z}\right) .
$$

Since $\beta_{Z}$ is the image of $\beta_{g(Z)}$ under the map $K_{m-2 n}^{M}(k(g(Z))) \rightarrow K_{m-2 n}^{M}(k(Z))$,

$$
N_{k(Z) / k(g(Z))}\left(\beta_{Z}\right)=m_{Z} \cdot \beta_{g(Z)} .
$$

Therefore $\psi_{Y} g_{*}(Z)=g_{*}^{\prime} \psi_{X}(Z)$.

Remark 1. In [21], Langsburg defined a map from $\mathbb{Z}_{X}^{c}(n)(X)$ to $C_{X}^{\mathrm{M}}(n)$ exactly the same as the one in Definition 2.14, except in case 3), instead of using $\beta_{Z}$, he used

$$
\beta_{Z}^{\prime}=\left\{\frac{t_{0}}{t_{m-2 n}}, \ldots, \frac{t_{m-2 n-1}}{t_{m-2 n}}\right\} .
$$

By multilinearity of Milnor K-theory, it is easy to see that $\beta_{Z}=\beta_{Z}^{\prime}$ up to 2-torsion element. Therefore, the map $\psi$ is equal to Langdsburg's map up to a 2-torsion. The advantage of using $\beta_{Z}$ is that one can use the results in [29]. On the other hand, using the idea in Landsburg's proof of showing that his map is a map of complexes, together with properties of $\chi$ in [29], we can give a direct proof of showing that $\psi$ is a map of complexes. This proof is lengthy, comparing to the one we give in Theorem 2.15. (There is a small gap in 
Landsburg's proof, as he only checks compatibility of his map with the differentials in the case of discrete valuation rings).

Remark 2. It is easy to see that the map $\psi$ can be generalized to define a map from Bloch's cycle complex $\mathbb{Z}_{X}^{c}(n)(X)$ to the corresponding cycle complex with coefficients in Milnor K-groups or Quillen K-groups defined by M. Rost [31].

Theorem 2.16. For any $X$ separated and essentially of finite type over $k$ of characteristic $p>0$, the map $\psi$ induces a quasi-isomorphism

$$
\hat{\psi}: \mathbb{Z}_{X}^{c} / p^{r}(n) \rightarrow \widetilde{\nu}_{X, r}(n) .
$$

Proof. Composing $\psi / p^{r}$ with the isomorphism

$$
C_{X}^{\mathrm{M}}(n) / p^{r} \stackrel{\sim}{\longrightarrow} \widetilde{\nu}_{X, r}(n)(X)
$$

we get a map of complexes

$$
\hat{\psi}: \mathbb{Z}_{X}^{c} / p^{r}(n)(X) \rightarrow \widetilde{\nu}_{X, r}(n)(X)
$$

To compare the cohomology of $\mathbb{Z}_{X}^{c} / p^{r}(n)$ and $\widetilde{\nu}_{X, r}(n)$, consider the niveau spectral sequence of higher Chow groups:

$$
E_{s, t}^{1}=\bigoplus_{X_{(s)}} H_{s+t}^{\mathrm{BM}}\left(k(x), \mathbb{Z} / p^{r}(n)\right) \Rightarrow H_{s+t}^{\mathrm{BM}}\left(X, \mathbb{Z} / p^{r}(n)\right) .
$$

By [14, Theorem 1.1], this spectral sequence collapses to give edge isomorphisms

$$
\Gamma: H_{s}^{\mathrm{BM}}\left(X, \mathbb{Z} / p^{r}(n)\right) \cong H_{s}\left(C_{X}^{\mathrm{HC}}(n) / p^{r}\right) .
$$

Composing with the isomorphisms

$$
C_{X}^{\mathrm{HC}}(n) / p^{r} \cong C_{X}^{\mathrm{M}}(n) / p^{r}(\text { Lemma2.12) }
$$

and

$$
C_{X}^{\mathrm{M}}(n) / p^{r} \cong \widetilde{\nu}_{X, r}(n)(X)(\operatorname{Lemma} 2.10),
$$

we get an isomorphism

$$
\hat{\Gamma}: H_{s}^{\mathrm{BM}}\left(X, \mathbb{Z} / p^{r}(n)\right) \cong H_{s}\left(\widetilde{\nu}_{X, r}(n)(X)\right) .
$$

Since $\phi$ induces edge morphisms of the spectral sequence, and $\psi=\chi \circ \phi$, we see that $\hat{\psi}$ induces $\hat{\Gamma}$. Hence $\hat{\psi}$ is a quasi-isomorphism.

$\mathbb{Q}$-coefficients. Let $k$ be a finite field with characteristic $p$ and $X$ be smooth and projective over $k$. Let $\mathbb{Q}_{X}^{c}(n)=\mathbb{Z}_{X}^{c}(n) \otimes \mathbb{Q}$. In [12, Proposition 2.1], conjecture $\mathrm{A}(n)$ (part of Parshin's conjecture) is equivalent to that, in the niveau spectral sequence

$$
E_{s, t}^{1}=\bigoplus_{X_{(s)}} H_{s+t}^{\mathrm{BM}}(k(x), \mathbb{Q}(n)) \Rightarrow H_{s+t}^{\mathrm{BM}}(X, \mathbb{Q}(n)),
$$

$E_{s, t}^{1}=0$ for $t \neq n$. In other words, it is equivalent to the existence of the following isomorphism:

$$
H_{s}\left(E_{*, n}^{1}\right) \cong H_{s}^{\mathrm{BM}}(X, \mathbb{Q}(n))
$$

Here $E_{*, n}^{1}$ is the Gersten complex of higher Chow groups with $\mathbb{Q}$-coefficients:

$$
\bigoplus_{X_{(d)}} H_{d+n}^{\mathrm{BM}}(k(x), \mathbb{Q}(n)) \rightarrow \ldots \rightarrow \bigoplus_{X_{(1)}} H_{1+n}^{\mathrm{BM}}(k(x), \mathbb{Q}(n)) \rightarrow \bigoplus_{X_{(0)}} H_{n}^{\mathrm{BM}}(k(x), \mathbb{Q}(n)) .
$$

Similar to the case above for the $\mathbb{Z} / p^{r}$-coefficients, we obtain the following theorem: 
Theorem 2.17. For smooth and projective varieties over finite fields, conjecture $A(n)$ is true if and only if $\phi$ induces a quasi-isomorphism from $\mathbb{Q}_{X}^{c}(n)(X)$ to the Gersten complex of higher Chow groups $E_{*, n}^{1}$ in $\mathbb{Q}$-coefficients .

\section{Comparison between Bloch's Complex and Spiess' Complex}

In this section, we assume that $S=\operatorname{Spec} k$ or $\operatorname{Spec} D$, and we will deal with the complex $\mathbb{Z}_{X}^{c}=\mathbb{Z}_{X}^{c}(0)$. We assume that $X$ is of finite type over $S$ and $\operatorname{dim}_{S} X=2$. Spiess's dualizing complex $\mathcal{K}_{X}$ of étale sheaves for surfaces uses the weight-two motivic complex defined by S. Lichtenbaum [24, Definition 2.1]. For a regular Noetherian ring $A$, let

$$
W=\operatorname{Spec} A[T], Z=\operatorname{Spec} A[T] / T(T-1),
$$

and $B=\left\{b_{1}, \ldots, b_{m}\right\}$ a finite set of exceptional units of $A$ (i.e., $b_{i}$ and $1-b_{i}$ are units).

Let

$$
Y_{B}=\operatorname{Spec} A[T] / \prod_{i=1}^{m}\left(T-b_{i}\right) .
$$

There is an exact sequence induced from relative K-theory

$$
K_{3}(A) \rightarrow K_{2}\left(W-Y_{B}, Z\right) \stackrel{\phi_{A, B}}{\longrightarrow} K_{1}^{\prime}\left(Y_{B}\right) \stackrel{\omega_{A, B}}{\longrightarrow} K_{2}(A) .
$$

Here $K_{i}^{\prime}$ is the K-theory of the category of quasi-coherent sheaves. Taking limits among all the $B$ 's, we obtain an exact sequence

$$
K_{3}(A) \rightarrow C_{2,1}(A) \stackrel{\phi_{A}}{\longrightarrow} C_{2,2}(A) \stackrel{\omega_{A}}{\longrightarrow} K_{2}(A)
$$

with

$$
C_{2,1}(A)=\underline{\lim _{\longrightarrow}} K_{2}\left(W-Y_{B}, Z\right), C_{2,2}(A)=\underline{\lim _{\longrightarrow}} K_{1}^{\prime}\left(Y_{B}\right) .
$$

If $X$ is a regular Noetherian scheme, associating the group $C_{2, i}(A)$ to each regular affine scheme $U=\operatorname{Spec} A$ which is étale over $X$, we obtain a presheaf on $X_{\text {ét. Denote by }}$ $\underline{C}_{2, i}(X)$ the associated étale sheaf. Then Lichtenbaum's weight-two motivic complex $\mathbb{Z}(2, X)$ is defined as the complex

$$
\underline{C}_{2,1}(X) \stackrel{\phi \times}{\rightarrow} \underline{C}_{2,2}(X)
$$

with the terms in cohomological degree 1 and 2, respectively. If $A=k$ is a field, there is an exact sequence [24, §3],

$$
K_{3}(k) \rightarrow C_{2,1}(k) \stackrel{\phi_{k}}{\longrightarrow} C_{2,2}(k) \stackrel{\omega_{k}}{\longrightarrow} K_{2}(k) \rightarrow 0 .
$$

Definition 3.1 (Spiess, [34, Definition 1.2.1]). Define the complex of étale sheaves $\mathcal{K}_{X}$ as follows:

$$
\bigoplus_{X_{(2)}} i_{x *} \underline{C}_{2,1}(k(x)) \stackrel{c_{3}}{\rightarrow} \bigoplus_{X_{(2)}} i_{x *} \underline{C}_{2,2}(k(x)) \stackrel{c_{2}}{\rightarrow} \bigoplus_{X_{(1)}} i_{x *} \mathbb{G}_{m} \stackrel{c_{1}}{\rightarrow} \bigoplus_{X_{(0)}} i_{x *} \mathbb{Z} .
$$

Here $c_{3}=\phi_{k(x)}, c_{1}$ is the map sending a rational function to its associated divisors, and $c_{2}$ is the composition

$$
\bigoplus_{X_{(2)}} i_{x *} \underline{C}_{2,2}(k(x)) \stackrel{\omega_{k(x)}}{\longrightarrow} \bigoplus_{X_{(2)}} i_{x *} K_{2}(k(x)) \stackrel{\partial^{\prime}}{\rightarrow} \bigoplus_{X_{(1)}} i_{x *} \mathbb{G}_{m},
$$

with $\partial^{\prime}$ the map in the Gersten resolution of $K$-theory [30, §7]. The terms are put in (cohomological) degree -3, -2, -1, 0, respectively.

Note that the assumption on degrees are different from that in [34]. 
Theorem 3.2 (Spiess, [34, Theorem 2.2.2]). For $X$ an equidimensional surface over $\mathbb{Z}$ satisfying the $(N R)$ condition and every constructible sheaf $\mathcal{F}$ on $X$, there are perfect pairings of finite groups

$$
H_{c}^{i}\left(X_{\hat{e}}, \mathcal{F}\right) \times \operatorname{Ext}_{X}^{2-i}\left(\mathcal{F}, \mathcal{K}_{X}\right) \rightarrow \mathbb{Q} / \mathbb{Z} .
$$

Here we say that $X$ satisfies the (NR) condition if

(NR): $k(x)$ is not formally real for every $x \in X$.

Theorem 3.3 (Spiess, [34, Proposition 2.3.2]). Let $k$ be an algebraically closed field of characteristic $p, X$ be an irreducible surface over $k$ and $\mathcal{F}$ be an $n$-torsion constructible sheaf on $X$, with $(n, p)=1$. Let $\mathcal{K}_{X}(n)=R H \mathrm{Rm}_{X}\left(\mathbb{Z} / n, \mathcal{K}_{X}\right)$. Then there are perfect pairings of finitely generated groups:

$$
H_{c}^{i}\left(X_{\text {et }}, \mathcal{F}\right) \times \operatorname{Ext}_{X}^{1-i}\left(\mathcal{F}, \mathcal{K}_{X}(n)\right) \rightarrow \mathbb{Z} / n .
$$

In [7], Deninger considered a dualizing complex of étale sheaves

$$
\mathcal{G}_{Y}: 0 \rightarrow \bigoplus_{Y_{(1)}} i_{y *} \mathbb{G}_{m} \rightarrow \bigoplus_{Y_{(0)}} i_{y, *} \mathbb{Z} \rightarrow 0
$$

for curve $Y$, and E. Nart [28] compared it with cycle complex $\mathbb{Z}_{Y}^{c}$ by constructing a map from $\mathbb{Z}_{Y}^{c}$ to $\mathcal{G}_{Y}$ which induces a quasi-isomorphism. Here we put the terms in (cohomological) degree -1 and 0 respectively. In what follows we generalize this method and define a similar complex for surfaces.

Consider the niveau spectral sequence of higher Chow groups for surfaces

$$
E_{s, t}^{1}=\bigoplus_{X_{(s)}} H_{s+t}^{\mathrm{BM}}(k(x), \mathbb{Z}) \Rightarrow H_{s+t}^{\mathrm{BM}}(X, \mathbb{Z}) .
$$

Only $E_{0,0}^{1}, E_{1,0}^{1}$ and $E_{2, t}^{1}(t \geq 0)$ are non-vanishing,

$$
H_{s+2}^{\mathrm{BM}}(X, \mathbb{Z}) \cong \bigoplus_{X_{(2)}} H_{s+2}^{\mathrm{BM}}(k(x), \mathbb{Z})
$$

for $s>0$, and in the bottom of the spectral sequence, the only nonvanishing terms in $E_{*, 0}^{1}$ are:

$$
\bigoplus_{X_{(2)}} H_{2}^{\mathrm{BM}}(k(x), \mathbb{Z}) \stackrel{f}{\rightarrow} \bigoplus_{X_{(1)}} k(x)^{*} \stackrel{d_{1}}{\rightarrow} \bigoplus_{X_{(0)}} \mathbb{Z}
$$

with

$$
\operatorname{coker} d_{1} \cong H_{0}^{\mathrm{BM}}(X, \mathbb{Z}), \frac{\operatorname{ker} d_{1}}{\operatorname{Im} f} \cong H_{1}^{\mathrm{BM}}(X, \mathbb{Z}), \operatorname{ker} f \cong H_{2}^{\mathrm{BM}}(X, \mathbb{Z})
$$

Definition 3.4. We define a cochain complex of abelian groups $\mathcal{C}(X)$ :

$$
\bigoplus_{X_{(2)}} \frac{z_{0}(k(x), 3)}{I_{x}} \stackrel{d_{3}}{\longrightarrow} \bigoplus_{X_{(2)}} z_{0}(k(x), 2) \stackrel{d_{2}}{\longrightarrow} \bigoplus_{X_{(1)}} k(x)^{*} \stackrel{d_{1}}{\longrightarrow} \bigoplus_{X_{(0)}} \mathbb{Z}
$$


Here $I_{x}=\operatorname{Im}\left(d_{x}: z_{0}(k(x), 4) \rightarrow z_{0}(k(x), 3)\right), d_{3}$ is the map induced by $g_{x}: z_{0}(k(x), 3) \rightarrow$ $z_{0}(k(x), 2), d_{1}=c_{1}$, and $d_{2}$ is the composition

$$
\bigoplus_{X_{(2)}} z_{0}(k(x), 2) \stackrel{\pi}{\longrightarrow} \bigoplus_{X_{(2)}} H_{2}^{\mathrm{BM}}(k(x), \mathbb{Z})
$$

The (cohomological) degrees of the terms are $-3,-2,-1,0$, respectively.

Theorem 3.5. Let $X$ be a surface over $\operatorname{Spec} k$ or $\operatorname{Spec} D$, then there is a map

$$
\psi^{\prime}: \mathbb{Z}_{X}^{c}(X) \rightarrow \mathcal{C}(X)
$$

which induces isomorphisms

$$
H^{-i}\left(\mathbb{Z}_{X}^{c}(X)\right) \cong H^{-i}(\mathcal{C}(X))
$$

for $0 \leq i \leq 3$.

Proof. Following the same idea of the definition of $\psi$ in $\S 2$, we can define a map from $\tau_{\geq-3} \mathbb{Z}_{X}^{c}(X)$ to $\mathcal{C}(X)$ :

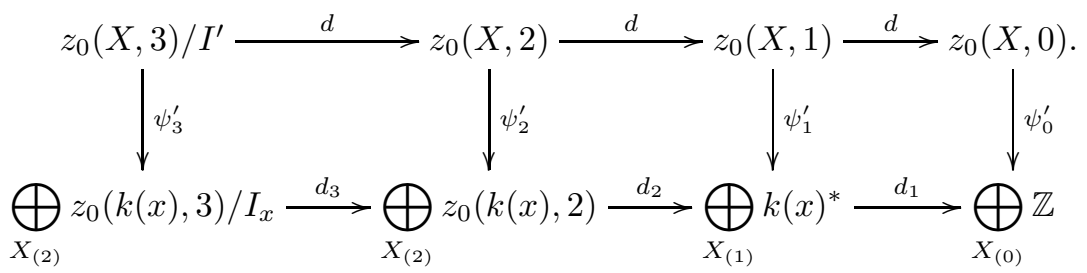

Here

$$
I^{\prime}=\operatorname{Im}\left(z_{0}(X, 4) \rightarrow z_{0}(X, 3)\right), \psi_{0}^{\prime} \stackrel{\text { def }}{=} \psi_{0}, \psi_{1}^{\prime} \stackrel{\text { def }}{=} \psi_{1} .
$$

For $i=2,3, \psi_{i}^{\prime}$ is defined as follows: if $\operatorname{dim} p(Z) \leq 1, \psi_{i}^{\prime}(Z)=0$; if $\operatorname{dim} p(Z)=2$, then $Z$ is dominant over some $x \in X_{(2)}$, so pulling back $Z$ along $\operatorname{Spec} k(x) \rightarrow X$, we get an element $\psi_{i}^{\prime}(Z) \in z_{0}(k(x), i)$. By similar argument as in $\S 2$, we see that the diagram is commutative. Moreover, the $\psi_{i}^{\prime}$ 's induce the corresponding isomorphisms from the degeneration of the niveau spectral sequence:

$$
\begin{gathered}
H_{0}^{\mathrm{BM}}(X, \mathbb{Z}) \cong \operatorname{coker} d_{1} \cong H_{0}(\mathcal{C}(X)), \\
H_{1}^{\mathrm{BM}}(X, \mathbb{Z}) \cong \frac{\operatorname{ker} d_{1}}{\operatorname{Im} f} \stackrel{(*)}{=} \frac{\operatorname{ker} d_{1}}{\operatorname{Im} d_{2}}=H_{1}(\mathcal{C}(X)), \\
H_{3}^{\mathrm{BM}}(X, \mathbb{Z}) \cong \bigoplus_{X_{(2)}} H_{3}^{\mathrm{BM}}(k(x), \mathbb{Z}) \cong \operatorname{ker} d_{3} \cong H_{3}(\mathcal{C}(X)), \\
H_{2}^{\mathrm{BM}}(X, \mathbb{Z}) \cong \operatorname{ker} f .
\end{gathered}
$$

Here the equality $(*)$ follows from the diagram 3.0.14).

It remains to show that $\operatorname{ker} f \cong H_{2}(\mathcal{C}(X))$. Look at the diagram (3.0.14). There is an exact sequence

$$
0 \rightarrow \operatorname{ker} \pi \rightarrow \operatorname{ker} d_{2} \rightarrow \operatorname{ker} f \rightarrow \operatorname{coker} \pi \rightarrow \operatorname{coker} d_{2} \rightarrow \operatorname{coker} f \rightarrow 0 .
$$


Since $\pi$ is surjective, coker $\pi=0$, so $\operatorname{ker} f \cong \operatorname{ker} d_{2} / \operatorname{ker} \pi$. But $\operatorname{ker} \pi=\operatorname{Im} d_{3}$. Hence

$$
H_{2}^{\mathrm{BM}}(X, \mathbb{Z}) \cong \operatorname{ker} f \cong \operatorname{ker} d_{2} / \operatorname{Im} d_{3} \cong H_{2}(\mathcal{C}(X)) \text {. }
$$

Remark. In the above proof, $\psi_{1}$ is similar to the map defined by Nart ([28]). As noted by Nart, there are only two types of generators in $z_{0}(X, 1)$, the vertical ones and the horizontal ones. The vertical ones are those $Z$ 's with $\operatorname{dim} p(Z)=0$, and the horizontal ones are those $Z$ 's with $p(Z)=\overline{\{x\}}$ for some $x \in X_{(1)}$. Nart's map sends the first type to 0 and the second type to $N\left(\frac{t_{0}}{t_{1}}\right)$ with $N: k(Z)^{*} \rightarrow k(x)^{*}$ the norm map of fields. In our situation, according to the Nesterenko-Suslin isomorphism, $\psi_{1}$ sends the first type to 0 and the second type to $N\left(\frac{-t_{0}}{t_{1}}\right)$. So it agrees with Nart's map up to 2-torsion.

Theorem 3.6. For a surface $X$ over $\operatorname{Spec} k$ or $\operatorname{Spec} D, \mathcal{C}(X) \cong \mathcal{K}_{X}(X)$ in the derived category of étale sheaves.

Before proving the theorem, we need the following lemma. First, let us recall some notations from [5]. From now on until Theorem 3.8, assume that $F$ is a field with $\operatorname{dim}_{S} F=$ 2. Let $\Delta^{p}=\Delta_{S}^{p}$. A closed subvariety

$$
\sigma: t_{i 1}=t_{i 2}=\ldots=t_{i q}=0
$$

is called a codimension $q$ face. A closed subvariety $V \subset \Delta^{p}$ is said to be in good position if $V \cap \sigma$ has codimension $\geq q$ in $V$ for any codimension $q$ face $\sigma$. Let

$$
\mathcal{V}^{n}=\mathcal{V}^{n}\left(\Delta^{p}\right) \subset \Delta^{p}
$$

denote the union of all codimension $n$ closed subvarieties of $\Delta^{p}$ in good position. Given a scheme $X$, we write $K(X)$ for some space, contravariant functorial in $X$, whose homotopy groups calculate the Quillen K-theory of $X$. For $Y \subset X$ a closed subset, we write $K(X, Y)$ for the homotopy fibre of $K(X) \rightarrow K(Y)$. This construction can be iterated. Given $Y_{1}, \ldots, Y_{n} \subset X$, we define the multi-relative K-space

$$
\begin{gathered}
K\left(X ; Y_{1}, \ldots, Y_{n}\right) \stackrel{\text { def }}{=} \text { homotopy fibre of } \\
\left(K\left(X ; Y_{1}, \ldots, Y_{n-1}\right) \rightarrow K\left(Y_{n} ; Y_{1} \cap Y_{n}, \ldots, Y_{n-1} \cap Y_{n}\right)\right) .
\end{gathered}
$$

Let

$$
\begin{gathered}
K\left(\Delta^{p}, \partial\right)=K\left(\Delta^{p} ; \partial_{0}, \ldots, \partial_{p}\right) \\
K\left(\Delta^{p}, \sum\right)=K\left(\Delta^{p} ; \partial_{0}, \ldots, \partial_{p-1}\right),
\end{gathered}
$$

where $\partial_{i}=\partial_{i}\left(\Delta^{p-1}\right)$. Let $\Psi=\partial$ or $\sum$. Define

$$
K_{V}\left(\Delta^{p}, \Psi\right) \stackrel{\text { def }}{=} \text { homotopy fibre of }\left(K\left(\Delta^{p}, \Psi\right) \rightarrow K\left(\Delta^{p}-V, \Psi-V\right)\right) .
$$

If $W \subset V$ is an inclusion of closed subvarieties, then there is a canonical map

$$
K_{W}\left(\Delta^{p}, \Psi\right) \rightarrow K_{V}\left(\Delta^{p}, \Psi\right)
$$

Hence we can define

$$
\begin{gathered}
K_{\mathcal{V}^{n}}\left(\Delta^{p}, \Psi\right) \stackrel{\text { def }}{=} \underset{V \subset \mathcal{V}^{n}}{\lim _{V}} K_{V}\left(\Delta^{p}, \Psi\right) \\
K_{\mathcal{V}^{n}-\mathcal{V}^{n+1}}\left(\Delta^{p}, \Psi\right) \stackrel{\text { def }}{=} \underset{V \subset \mathcal{l i m}_{W}}{\underset{\subset \mathcal{V}^{n+1}}{\lim }} K_{V-W}\left(\Delta^{p}-W, \Psi-W\right) .
\end{gathered}
$$


There are two isomorphisms from $H_{2}^{\mathrm{BM}}(F, \mathbb{Z})$ to $K_{2}(F)$ in [5]: the first one $\theta^{\prime}$ is induced by the edge morphisms of the spectral sequence

$$
E_{2}^{p, q}=H_{q-p+4}^{\mathrm{BM}}(F, \mathbb{Z}(2+q)) \Rightarrow K_{-p-q}(F)
$$

proved in [5] (note that we have changed the notation in loc. it. to the motivic Borel-Moore homology of $F$ over base $S$ ); the second one $\theta$ is induced from the quasi-isomorphism between $T_{F}$ and $\mathbb{Z}(2, F)$ (loc.it., $\left.\S 7\right)$. Here $T_{F}$ is the following truncation of cycle complex of fields:

$$
z_{0}(F, 3) / I_{F} \rightarrow z_{0}(F, 2)
$$

with

$$
I_{F} \stackrel{\text { def }}{=} \operatorname{Im}\left(z_{0}(F, 4) \rightarrow z_{0}(F, 3)\right) .
$$

Hence coker $T_{F}=H_{2}^{B M}(F, \mathbb{Z})$. From 3.0.11, we know that this quasi-isomorphism induces an isomorphism:

$$
\theta: H_{2}^{B M}(F, \mathbb{Z}) \rightarrow K_{2}(F) .
$$

Lemma 3.7. (1) For any field $F$,

$$
\theta=\theta^{\prime}: H_{2}^{B M}(F, \mathbb{Z}) \rightarrow K_{2}(F) .
$$

(2) The following diagram is commutative

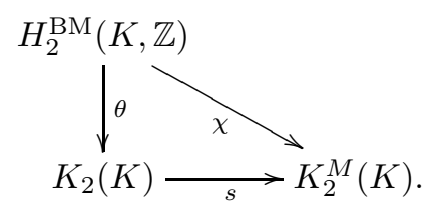

Proof. (1) $\Rightarrow(2)$ : By [14, Proposition 3.3], we know that $s \circ \chi=\theta^{\prime}$. Then from (1), we have

$$
s \circ \chi=\theta^{\prime}=\theta .
$$

(1) By the definitions of $\theta$ and $\theta^{\prime}$ [5, $\S 1$ and $\S 7$, and the following isomorphisms:

$$
K_{0}\left(\Delta^{2}, \partial\right) \cong K_{1}\left(\Delta^{1}, \partial\right) \stackrel{\cong}{\leftrightarrows} K_{2}\left(\Delta^{0}\right),
$$

we see that the two maps are induced by the two paths from $H_{2}^{\mathrm{BM}}(F, \mathbb{Z})$ to $K_{1}\left(\Delta^{1}, \partial\right)$ and to $K_{0}\left(\Delta^{2}, \partial\right)$ shown in the following diagram:

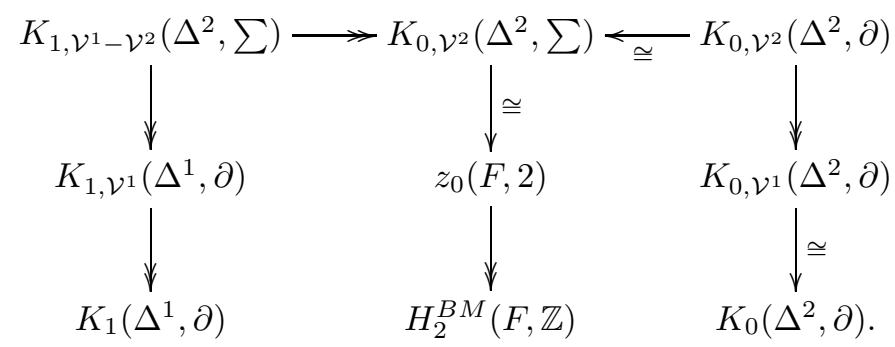

The map $\theta^{\prime}$ is the path from $z_{0}(F, 2)$ to $K_{0, \mathcal{V}^{2}}\left(\Delta^{2}, \partial\right)$ and then to $K_{0}\left(\Delta^{2}, \partial\right)$, while $\theta$ is the path from $z_{0}(F, 2)$ to $K_{1}\left(\Delta^{1}, \partial\right)$ via $K_{1, \mathcal{V}^{1}-\mathcal{V}^{2}}\left(\Delta^{2}, \sum\right)$. In order to show that $\theta=\theta^{\prime}$, it 
suffices to show commutativity of the following diagram: (starting from $K_{1, \mathcal{V}^{1}-\mathcal{V}^{2}}\left(\Delta^{2}, \sum\right.$ ) and end at $\left.K_{0}\left(\Delta^{2}, \partial\right)\right)$

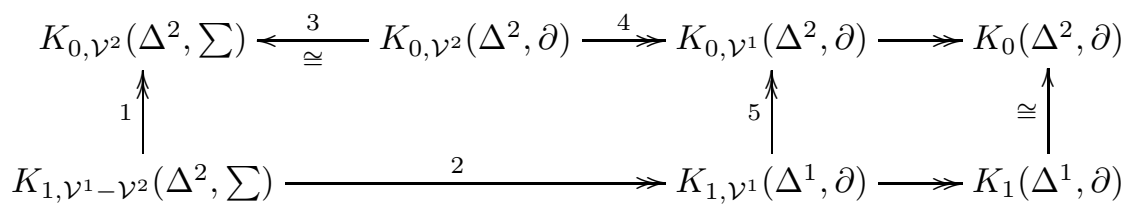

Here the right hand square is induced from the embedding $\left(\Delta^{1}, \partial_{0}, \partial_{1}\right) \subset\left(\Delta^{2}, \partial_{0}, \partial_{1}\right)$, so it is commutative. Therefore we have to show that the square on the left commutes, or more precisely, $5 \circ 2=4 \circ 3^{-1} \circ 1$.

Recall the long exact sequence of relative K-groups:

$$
K_{1}(Y) \stackrel{i}{\rightarrow} K_{0}(X, Y) \stackrel{j}{\rightarrow} K_{0}(X) \stackrel{k}{\rightarrow} K_{0}(Y) .
$$

That is the only type of long exact sequence involved in the diagrams above. In particular,

$$
1 \sim i, 2 \sim k, 3 \sim j, 4 \sim k, 5 \sim i .
$$

Here map 1 is for the embedding $\left(\Delta^{2}-\mathcal{V}^{1}, \sum\right) \subset\left(\Delta^{2}-\mathcal{V}^{2}, \sum\right)$, map 4 is for the embedding $\left(\Delta^{2}-\mathcal{V}^{1}, \partial\right) \subset\left(\Delta^{2}-\mathcal{V}^{2}, \partial\right)$, maps 2,3 and 5 are for the embedding $\left(\Delta^{1}, \partial\right) \subset$ $\left(\Delta^{2}, \sum\right)$ (embedding of the last face), and $1 \sim i$ means that the map 1 corresponds to type $i$ in the sequence (3.0.17), etc. By functoriality of relative sequence of K-theory, we obtain the commutativity.

Proof of Theorem 3.6. Let us first define a map $\mathcal{C}(X) \rightarrow \mathcal{K}_{X}(X)$ in the derived category. For any $x \in X_{(2)}$, there is a quasi-isomorphism [5, Theorem 7.2] in the derived category between the complex

$$
z_{0}(k(x), 3) / I_{x} \rightarrow z_{0}(k(x), 2)
$$

and the complex

$$
C_{2,1}(k(x)) \rightarrow C_{2,2}(k(x)) .
$$

Moreover, it induces an isomorphism between the cokernels of the two complexes

$$
H_{2}^{\mathrm{BM}}(k(x), \mathbb{Z}) \stackrel{\theta_{x}}{\sim} K_{2}(k(x))
$$

(see (3.0.16) above). Recall that the complex $\mathcal{C}(X)$ (resp., $\left.\mathcal{K}_{X}(X)\right)$ is defined by connecting

$$
\begin{gathered}
\bigoplus_{X_{(2)}}\left(z_{0}(k(x), 3) / I_{x}\right) \stackrel{d_{3}}{\longrightarrow} \bigoplus_{X_{(2)}} z_{0}(k(x), 2) \\
\text { (resp., } \left.\bigoplus_{X_{(2)}} C_{2,1}(k(x)) \stackrel{c_{3}}{\rightarrow} \bigoplus_{X_{(2)}} C_{2,2}(k(x))\right)
\end{gathered}
$$

with

$$
\bigoplus_{X_{(1)}} k(x)^{*} \stackrel{d_{1}}{\longrightarrow} \bigoplus_{X_{(0)}} \mathbb{Z}
$$


via $\oplus_{X_{(2)}} H_{2}^{\mathrm{BM}}(k(x), \mathbb{Z})$ (resp., $\left.\oplus_{X_{(2)}} K_{2}(k(x))\right)$. Since $c_{1}=d_{1}$, it suffices to show that the following diagram is (anti-)commutative:

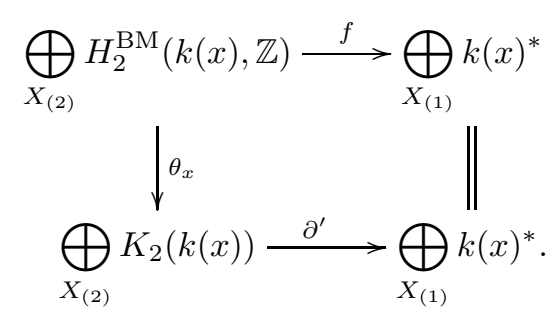

Let $\eta \in X_{(2)}$ and $K=k(\eta)$, consider the following diagram:

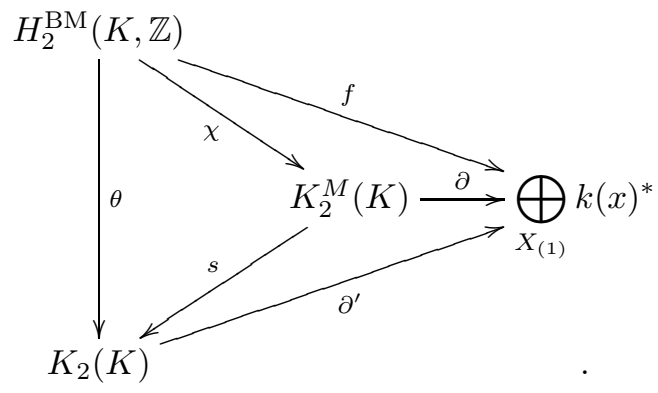

Here $\partial$ is the tame symbol and $\chi$ is the Nesterenko-Suslin isomorphism. We have to show that the outside triangle is (anti-)commutative, so it suffices to show that the three small triangles are (anti-)commutative. The triangle on the top is commutative by Lemma 2.12. The one on the left commutes by Lemma 3.7

For the triangle on the bottom, we have to show commutativity of the following diagram:

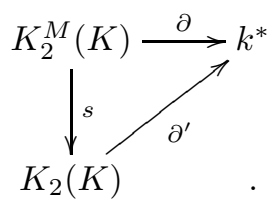

Here $k$ is the residue field of a valuation $v$ of $K$, with $\pi$ a prime element. From [31, Definition $1.1 \mathrm{R} 3 \mathrm{e}], \partial^{\prime}$ has the following property: for any $\rho, u \in K^{*}$ with $v(u)=0$, $\partial^{\prime}(\{u\} \cdot \rho)=-\{\bar{u}\} \cdot v(\rho)$, i.e. $\partial^{\prime} \circ s(\{u, \rho\})=\{\bar{u}\}^{-v(\rho)}$. Here $\bar{u}$ is the residue class of $u$ in $k^{*}$. But

$$
\left.\partial(\{u, \rho\})=(-1)^{v(u) v(\rho)} \overline{\left\{\frac{u^{v(\rho)}}{\rho^{v(u)}}\right.}\right\}=\{\bar{u}\}^{v(\rho)} .
$$

By multilinearity of Milnor K-theory, any element $\left\{u_{1} \pi^{n}, u_{2} \pi^{m}\right\}$ with $v\left(u_{i}\right)=0$ can be decomposed into a product of elements of the form $\{u, \pi\}$ and $\{\pi, \pi\}=\{-1, \pi\}$ with $v(u)=0$. Therefore, the diagram commutes up to sign. So the triangle outside is commutative. In conclusion, there is an morphism from $\mathcal{C}(X)$ to $\mathcal{K}_{X}$ in the derived category of étale sheaves.

Now let us compare the (co)homology groups. It is clear that

$$
H_{0}(\mathcal{C}(X)) \cong H_{0}\left(\mathcal{K}_{X}(X)\right) \text {. }
$$

From [5, Theorem 7.2],

$$
H_{3}(\mathcal{C}(X)) \cong H_{3}\left(\mathcal{K}_{X}(X)\right)
$$


To compare $c_{2}$ and $d_{2}$, consider the diagrams defining them

$$
\bigoplus_{X_{(2)}} z_{0}(k(x), 2) \stackrel{\pi}{\longrightarrow} \bigoplus_{X_{(2)}} H_{2}^{\mathrm{BM}}(k(x), \mathbb{Z}) \quad \bigoplus_{X_{(2)}} C_{2,2}(k(x)) \stackrel{\omega_{k(x)}}{\longrightarrow} \bigoplus_{X_{(2)}} K_{2}(k(x))
$$

Here $\pi$ and $\omega_{k(x)}$ are both surjective onto groups that are connected via the isomorphism

$$
\theta: H_{2}^{\mathrm{BM}}(k(x), \mathbb{Z}) \rightarrow K_{2}(k(x)),
$$

and $\partial^{\prime} \theta=f$, so

$$
\operatorname{Im} d_{2}=\operatorname{Im} f=\operatorname{Im} \partial^{\prime}=\operatorname{Im} c_{2}
$$

Therefore,

$$
H_{1}(\mathcal{C}(X)) \cong \operatorname{ker} d_{1} / \operatorname{Im} d_{2} \cong \operatorname{ker} c_{1} / \operatorname{Im} c_{2} \cong H_{1}\left(\mathcal{K}_{X}(X)\right) .
$$

Similar to the proof of Theorem 3.5 , we have that

$$
H_{2}(\mathcal{C}(X)) \cong \operatorname{ker} f \cong \operatorname{ker} \partial^{\prime} \cong H_{2}\left(\mathcal{K}_{X}(X)\right) \text {. }
$$

In the following theorem we will need to consider fields of dimension 2 over the base $S$. If $S=\operatorname{Spec} k$, a field $K$ of transcendental degree 2 over $S$ has $\operatorname{dim}_{S} K=2$. If $S=\operatorname{Spec} D$, the function field $K$ of a scheme $X$ with $\operatorname{dim}_{S} X=2$ will have $\operatorname{dim}_{S} K=2$.

Theorem 3.8. Suppose that $X$ is a two-dimensional scheme over $S=\operatorname{Spec} k$ or $S=$ Spec $D$ and the function field of $X$ is $K$. Then there is a map in the derived category $\mathbb{Z}_{X}^{c} \rightarrow \mathcal{K}_{X}$ which induces a quasi-isomorphism

$$
\tau_{\geq-3} \mathbb{Z}_{X}^{c} \cong \mathcal{K}_{X}
$$

Assuming the Beilinson-Soulé Conjecture for $\mathbb{Z}(2)$ on fields of dimension 2 over $S$, then $\mathbb{Z}_{X}^{c} \cong \mathcal{K}_{X}$ in the derived category. Here $\mathbb{Z}(n)$ is Voevodsky's motivic complex.

Proof. From Theorem 3.5 and 3.6 we know that in the derived category, there is a map $\mathbb{Z}_{X}^{c} \rightarrow \mathcal{K}_{X}$. Moreover,

$$
\tau_{\geq-3} \mathbb{Z}_{X}^{c} \cong \mathcal{K}_{X}
$$

The Beilinson-Soulé Conjecture [17, Conjecture 5] asserts that for a regular scheme $X$ and $n>0, H^{i}(X, \mathbb{Z}(n))=0$ for $i \leq 0$. In particular, for $\mathbb{Z}(2)$ on the function field $K$ of $X$ (note that $\operatorname{dim}_{S} K=2$ ), it is equivalent to asserting that

$$
H_{i}^{\mathrm{BM}}(K, \mathbb{Z})=H^{4-i}(K, \mathbb{Z}(2))=0
$$

for $i \geq 4$. To prove that $\mathbb{Z}_{X}^{c} \cong \tau_{\geq-3} \mathbb{Z}_{X}^{c}$, we need to show that $\mathbb{Z}_{X}^{c}(X)$ is acyclic at (cohomological) degree $\leq-4$. Now we have two cases, (i) $X$ over $S=\operatorname{Spec} k$, or $X$ over $S=\operatorname{Spec} D$ but factors through some closed point, (ii) $X$ flat over $S=\operatorname{Spec} D$.

case (i) This means that $X$ is a scheme over a perfect field $k$. Let $U \hookrightarrow X$ be any open subscheme of $X$ with complement $Z \hookrightarrow X$. Enlarge $Z$ so that it has dimension 1 . Then there is a long exact sequence

$$
\rightarrow H_{i}^{\mathrm{BM}}(Z, \mathbb{Z}) \rightarrow H_{i}^{\mathrm{BM}}(X, \mathbb{Z}) \rightarrow H_{i}^{\mathrm{BM}}(U, \mathbb{Z}) \rightarrow .
$$


By [28, Theorem 1.1], $\mathbb{Z}_{Z}^{c}(Z)$ is quasi-isomorphic to $\mathcal{G}_{Z}(Z)$ (Deninger's complex, see (3.0.12) $)$, so $H_{i}^{\mathrm{BM}}(Z, \mathbb{Z})=0$ for $i \geq 2$. Hence $H_{i}^{\mathrm{BM}}(X, \mathbb{Z}) \cong H_{i}^{\mathrm{BM}}(U, \mathbb{Z})$ for $i \geq 3$. Take limit among all the open subschemes over $X$, we get that, for $i \geq 3$,

$$
H^{-i}\left(\mathbb{Z}_{X}^{c}(X)\right)=H_{i}^{\mathrm{BM}}(X, \mathbb{Z}) \cong H_{i}^{\mathrm{BM}}(K, \mathbb{Z})=0 .
$$

Hence

$$
\mathbb{Z}_{X}^{c} \cong \tau_{\geq-3} \mathbb{Z}_{X}^{c} \cong \mathcal{K}_{X}
$$

case (ii) $X$ is flat over $S=\operatorname{Spec} D$. This is a local problem, so we can assume that $D$ is a discrete valuation ring. Let $U$ be the generic fiber and $Z$ be the closed fiber. Then $Z$ is a curve over the residue field of $D$. Similar as in $(i)$, we can get the conclusion.

Corollary 3.9. Suppose that $X$ is a two-dimensional scheme over $S=\operatorname{Spec} k$ or $S=$ Spec $D$. Then for any torsion sheaf $\mathcal{F}$ on $X$, there is an isomorphism

$$
\operatorname{RHom}_{X}\left(\mathcal{F}, \mathbb{Z}_{X}^{c}\right) \rightarrow \operatorname{RHom}_{X}\left(\mathcal{F}, \mathcal{K}_{X}\right) .
$$

Proof. First, we assume that the conclusion is true for constructible sheaves on surfaces smooth over $S=\operatorname{Spec} k$ or $\operatorname{Spec} D$. If $X$ is over $S=\operatorname{Spec} k$ and not smooth, and $\mathcal{F}$ is constructible, choose an open smooth subscheme $U \stackrel{j}{\rightarrow} X$ with complement $Z \stackrel{i}{\rightarrow} X$ of codimension 1. By purity, $R i^{\prime} \mathbb{Z}_{X}^{c} \cong \mathbb{Z}_{Z}^{c}$ [9, Proposition 3.5] and $R i^{\prime} \mathcal{K}_{X} \cong \mathcal{G}_{Z}$ [34, Proposition 1.3.1 (ii)]. From the short exact sequence

$$
0 \rightarrow j ! j^{*} \mathcal{F} \rightarrow \mathcal{F} \rightarrow i_{*} i^{*} \mathcal{F} \rightarrow 0,
$$

we obtain a commutative diagram of distinguished triangles

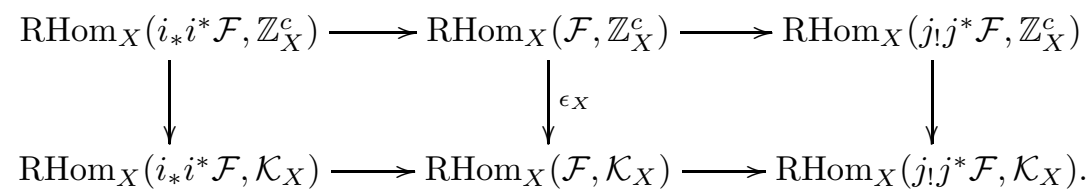

By adjointness and purity, the groups on the left are canonically isomorphic to

$$
\operatorname{RHom}_{Z}\left(i^{*} \mathcal{F}, \mathbb{Z}_{Z}^{c}\right) \text { and } \operatorname{RHom}_{Z}\left(i^{*} \mathcal{F}, \mathcal{G}_{Z}\right),
$$

respectively. Hence, by Nart [28], the left hand vertical map is an isomorphism. By adjointness, the groups on the right can be identified with

$$
\operatorname{RHom}_{U}\left(j^{*} \mathcal{F}, \mathbb{Z}_{U}^{c}\right) \text { and } \operatorname{RHom}_{U}\left(j^{*} \mathcal{F}, \mathcal{K}_{U}\right),
$$

respectively. Since $U$ is smooth, the right hand vertical map is an isomorphism by induction assumption. Therefore, the vertical map in the middle is an isomorphism.

Let $X$ be a scheme over $S=\operatorname{Spec} D$ but not smooth and $\mathcal{F}$ is constructible. If $X \rightarrow S$ factors through some closed point of $S$, then it is indeed a variety over a perfect field, in which case it is proved as above. If $X \rightarrow S$ does not factor through any closed point of $S$, then it is flat over $S$. It suffices to assume that $D$ is a discrete valuation ring. Let $U$ be the generic fiber and $Z$ be the special fiber, then $Z$ is a curve over a field. For the decomposition

$$
U \subset X \supset Z,
$$

we can apply the strategy as above to prove the conclusion for $X$ (see [9, Corollary 7.2] for purity of cycle complex).

For general torsion sheaf $\mathcal{F}$, it can be written as $\mathcal{F}=\operatorname{colim} \mathcal{F}_{i}$ with $\mathcal{F}_{i}$ constructible [26. Chapter II, Proposition 0.9]. So

$$
\operatorname{RHom}_{X}\left(\mathcal{F}_{i}, \mathbb{Z}_{X}^{c}\right) \cong \operatorname{RHom}_{X}\left(\mathcal{F}_{i}, \mathcal{K}_{X}\right) .
$$


Since for any sheaf $\mathcal{H}$,

$$
\operatorname{RHom}_{X}\left(\operatorname{colim} \mathcal{F}_{i}, \mathcal{H}\right) \cong \mathrm{R} \lim \operatorname{RHom}_{X}\left(\mathcal{F}_{i}, \mathcal{H}\right),
$$

the canonical map $\mathbb{Z}_{X}^{c} \rightarrow \mathcal{K}_{X}$ in the derived category induces a map

$$
\mathrm{R} \lim \operatorname{RHom}_{X}\left(\mathcal{F}_{i}, \mathbb{Z}_{X}^{c}\right) \rightarrow \mathrm{R} \lim \operatorname{RHom}_{X}\left(\mathcal{F}_{i}, \mathcal{K}_{X}\right),
$$

hence a map of spectral sequences

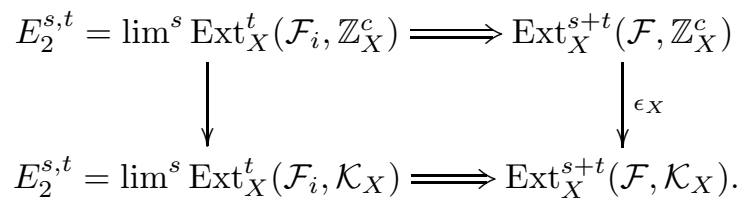

Note that these two spectral sequences are convergent. For the first one, it follows from 9 Corollary 4.9] (the statement in this Corollary is for Spec $k$-schemes, but the proof holds for Spec $D$-schemes). For the second one, since $\mathcal{K}_{X} \cong \tau_{\geq-3} \mathbb{Z}_{X}^{c}$, hence it is a bounded spectral sequence. The two spectral sequences are isomorphic at the $E_{2}$-page via maps defined by $\mathbb{Z}_{X}^{c} \rightarrow \mathcal{K}_{X}$, hence

$$
\operatorname{RHom}_{X}\left(\mathcal{F}, \mathbb{Z}_{X}^{c}\right) \cong \operatorname{RHom}_{X}\left(\mathcal{F}, \mathcal{K}_{X}\right) .
$$

Now let us prove the conclusion for constructible sheaves on surfaces smooth over $S$. Again, we separate the cases: a) $S=\operatorname{Spec} k$ with characteristic $p$; b) $S=\operatorname{Spec} D$.

a) Let $\mathcal{F}$ be a $\mathbb{Z} / p^{r}$-sheaf, with $p=\operatorname{char}(k)$. By the niveau spectral sequence of higher Chow groups with $\mathbb{Z} / p^{r}$ coefficients, for $t \geq 3$,

$$
H_{t}^{\mathrm{BM}}\left(X, \mathbb{Z} / p^{r}\right) \cong \bigoplus_{X_{(2)}} H_{t}^{\mathrm{BM}}\left(k(x), \mathbb{Z} / p^{r}\right) .
$$

By [14, Theorem 1.1], we see that $H_{t}^{\mathrm{BM}}\left(X, \mathbb{Z} / p^{r}\right)=0$ for $t \geq 3$. Therefore,

$$
\mathbb{Z}_{X}^{c} / p^{r} \cong \tau_{\geq-3} \mathbb{Z}_{X} / p^{r} \cong \mathcal{K}_{X} / p^{r} .
$$

In particular, for such $\mathcal{F}$,

$$
\epsilon_{X}: \operatorname{RHom}_{X}\left(\mathcal{F}, \mathbb{Z}_{X}^{c}\right) \stackrel{\cong}{\longrightarrow} \operatorname{RHom}_{X}\left(\mathcal{F}, \mathcal{K}_{X}\right) .
$$

Now let $\mathcal{F}$ be an $n$-torsion sheaf, with $(n, p)=1$. Let $\mathbb{Z} / n(2)=\mu_{n} \otimes \mu_{n}$. Since both $\mathbb{Z}_{X}^{c}$ and $\mathcal{K}_{X}$ satisfy the Kummer sequence (see [13, Theorem 1.5] and [34, Proposition 1.5.1], respectively):

thus,

$$
\begin{aligned}
& \rightarrow \mathbb{Z}_{X}^{c} \stackrel{n}{\rightarrow} \mathbb{Z}_{X}^{c} \rightarrow \mathbb{Z} / n(2) \rightarrow, \\
& \rightarrow \mathcal{K}_{X} \stackrel{n}{\rightarrow} \mathcal{K}_{X} \rightarrow \mathbb{Z} / n(2) \rightarrow,
\end{aligned}
$$

$$
\operatorname{RHom}_{X}\left(\mathcal{F}, \mathbb{Z}_{X}^{c}\right) \cong \operatorname{RHom}_{X}\left(\mathcal{F}, \mathcal{K}_{X}\right)
$$

b) Let $X$ be surface smooth over $\operatorname{Spec} D$. Let $\mathcal{F}$ be an $n$-torsion. If $n$ is invertible on $X$, then $\mathbb{Z}_{X}^{c}$ and $\mathcal{K}_{X}$ satisfy the Kummer sequence [34, Proposition 1.5.1]. Hence

$$
\epsilon_{X}: \operatorname{RHom}\left(\mathcal{F}, \mathbb{Z}_{X}^{c}\right) \rightarrow \operatorname{RHom}\left(\mathcal{F}, \mathcal{K}_{X}\right)
$$

is an isomorphism. If $n$ is not invertible on $X$, consider the following decomposition

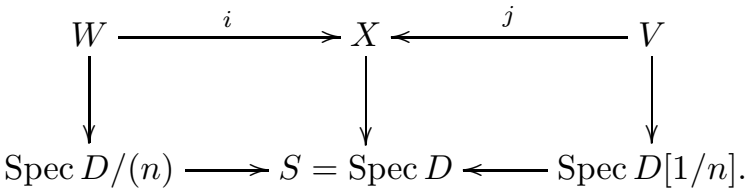


Here the two squares are fiber products. Then $i$ and $j$ are closed and open immersions, respectively. Since $X$ is flat over $S, W$ has codimension 1. Similar as in 3.0 .18 , there is a localization diagram and the vertical maps on the left and right are isomorphisms, which proves that

$$
\epsilon_{X}: \operatorname{RHom}_{X}\left(\mathcal{F}, \mathbb{Z}_{X}^{c}\right) \rightarrow \operatorname{RHom}_{X}\left(\mathcal{F}, \mathcal{K}_{X}\right)
$$

is an isomorphism. This finishes the proof.

\section{Comparison Between Bloch's Complex ANd SATO's CompleX}

Sato's complex $\mathfrak{I}_{r}(n)_{X}$. K. Sato [32] defined a complex $\mathfrak{I}_{r}(n)_{X}$ for certain schemes over Dedekind domains. Let us first recall his notations and definitions. In this section, $S=$ Spec $D$. Let $\Sigma$ be the set of closed points of $S=\operatorname{Spec} D$ of characteristic $p$. Suppose that $\Sigma \neq \emptyset$. Throughout this section, the scheme $X$ over $D$ is assumed to satisfy the following condition:

Condition 4.1 ([32], Condition 4.1.2). Let $X$ be a regular, pure-dimensional scheme of dimension d, flat and of finite type over $S$. For any $s \in \Sigma$, each connected component $X^{\prime}$ of

$$
X \times{ }_{S} \operatorname{Spec}\left(D_{s}^{h}\right)
$$

is a regular semi-stable family over $D_{s}^{h}$ or over the integral closure of $D_{s}^{h}$ in $\Gamma\left(X^{\prime}, \mathcal{O}_{X^{\prime}}\right)$. Here $D_{s}^{h}$ is the Hensenlization of $D$ at $s$, and a scheme flat and of finite type over a discrete valuation ring is called a regular semi-stable family if it is regular, its generic fiber is smooth, and its special fiber is a normal crossing divisor.

Let $Y \subset X$ be the divisor defined by the radical ideal $(p) \subset \mathcal{O}_{X}$. Let $U$ be its complement, and $\iota$ and $j$ be as follows:

$$
U \stackrel{j}{\longrightarrow} X \stackrel{\iota}{\longleftarrow} Y .
$$

Definition 4.2. Define $\nu_{Y, r}^{n}$ to be the étale sheaf on $Y$

$$
\nu_{Y, r}^{n} \stackrel{\text { def }}{=} \operatorname{ker}\left(\bigoplus_{y \in Y^{(0)}} i_{y *} \nu_{y, r}^{n} \stackrel{\partial}{\longrightarrow} \bigoplus_{Y^{(1)}} i_{y *} \nu_{y, r}^{n-1}\right) .
$$

Here $\partial$ is the map in the Gersten complex of logarithmic de Rham-Witt sheaves (see [20, $\S 1]$ or Lemma 2.10 above).

Define $M_{r}^{n}=\iota^{*} R^{n} j_{*} \mu_{p^{r}}^{\otimes n}$ on $Y$. We recall Sato's definition of the map

$$
\sigma_{X, r}^{n}: M_{r}^{n} \rightarrow \nu_{Y, r}^{n-1} .
$$

Since it is a map of sheaves, it can be defined on the stalks at points in $Y$, i.e., points of characteristic $p$. Let $y \in Y$ and $s \in \Sigma$ be its image in $S$. After replacing $D$ by $D_{s}^{h}$ and $X$ by $X \times_{S} \operatorname{Spec}\left(D_{s}^{h}\right)$ (and then replacing $D_{s}^{h}$ by its integral closure in one of the irreducible components of $X \times{ }_{S} \operatorname{Spec}\left(D_{s}^{h}\right)$, if necessary, depending the condition (4.1)), we can assume that $X$ is a regular semi-stable family over an Henselian discrete valuation ring $O_{K}$ with function field $K$ of characteristic 0 and residue field $k$ of characteristic $p$. Let

$$
i_{x}: x \rightarrow X_{K} \rightarrow X, i_{y}: y \rightarrow X_{k}
$$


be the embedding of points in $X_{K}$ and $X_{k}$, respectively. Then there is a diagram:

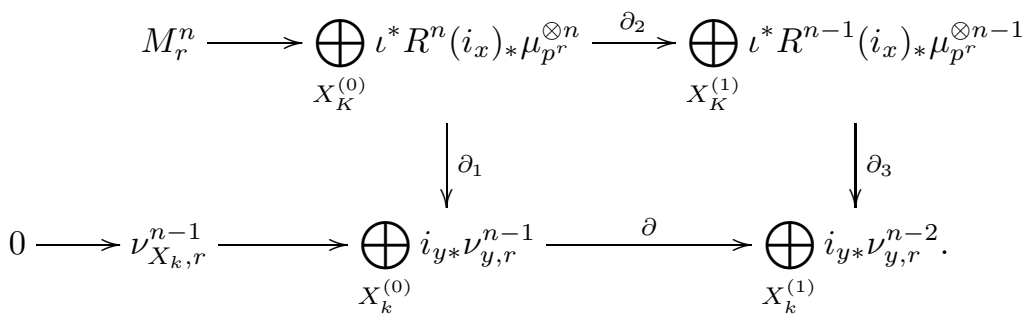

Here all the $\partial_{i}$ 's are boundary maps defined in [20, $\left.\S 1\right]$ (or see [32, Lemma 3.2.4]). The upper row is part of the Gersten complex of $\mu_{p^{r}}^{\otimes n}$ on $X$. The lower row is exact by definition. The square is anti-commutative by Kato, [20,1.7]. Hence it induces a map

$$
\sigma_{X, r}^{n}: M_{r}^{n} \rightarrow \nu_{X_{k}, r}^{n-1} .
$$

Define the map $\sigma_{X, r}(n)$ as the composition:

$$
\sigma_{X, r}(n): \tau_{\leq n} R j_{*} \mu_{p^{r}}^{\otimes n} \rightarrow\left(R^{n} j_{*} \mu_{p^{r}}^{\otimes n}\right)[-n] \stackrel{\sim}{\stackrel{\sim}{\diamond}}\left(\iota_{*} M_{r}^{n}\right)[-n] \stackrel{\sigma_{X, r}^{n}}{\longrightarrow} \iota_{*} \nu_{Y, r}^{n-1}[-n] .
$$

Here $\diamond$ is the adjunction $I d \rightarrow \iota_{*} \iota^{*}$.

Definition 4.3 (Sato, [32, Lemma 4.2.2]). If $n \geq 1$, and let $\mathfrak{I}_{r}(n)_{X}$ be a complex fitted into the following distinguished triangle

$$
\iota_{*} \nu_{Y, r}^{n-1}[-n-1] \stackrel{g}{\longrightarrow} \mathfrak{I}_{r}(n)_{X} \stackrel{t}{\longrightarrow} \tau_{\leq n} R j_{*} \mu_{p^{r}}^{\otimes n} \stackrel{\sigma_{X, r}(n)}{\longrightarrow} \iota_{*} \nu_{Y, r}^{n-1}[-n] .
$$

Then $\mathfrak{I}_{r}(n)_{X}$ is concentrated in $[0, n]$. Moreover, the triple $\left(\mathfrak{I}_{r}(n)_{X}, t, g\right)$ is unique up to a unique isomorphism and $g$ is determined by $\left(\mathfrak{I}_{r}(n)_{X}, t\right)$.

The duality theorem induced by the complex $\mathfrak{I}_{r}(n)_{X}$ is as follows

Theorem 4.4 (Sato, [32, Theorem 1.2.2]). Assume that $D$ is an number ring and $X$ is proper over $S=\operatorname{Spec} D$ satisfying Condition (4.1). $\operatorname{dim}_{S} X=d$. Then there is a perfect pairing of finite groups:

$$
H_{c}^{q}\left(X, \mathfrak{I}_{r}(n)_{X}\right) \times H_{e ́ t}^{2 d+1-q}\left(X, \mathfrak{I}_{r}(d-n)_{X}\right) \longrightarrow H_{c}^{2 d+1}\left(X, \mathfrak{I}_{r}(d)_{X}\right) \stackrel{\cong}{\longrightarrow} \mathbb{Z} / p^{r} .
$$

The following proposition and theorem compare $\mathfrak{I}_{r}(n)_{X}$ with Bloch's cycle complex, which partially proves a conjecture made by Sato [32, Conjecture 1.4.1]. If $X$ is smooth over $D$, the conclusion (without truncation) was proved by Geisser in [10, Theorem 1.3].

Proposition 4.5. Let $X$ satisfy Condition (4.1), then

(1) There is an isomorphism in $D^{b}\left(X_{e t}, \mathbb{Z} / p^{r}\right)$

$$
\mathfrak{I}_{r}(n)_{X} \stackrel{\cong}{\longrightarrow} \tau_{\leq n}\left(\mathbb{Z}_{X}^{c} / p^{r}(d-n)[-2 d]\right) .
$$

(2)There is an isomorphism in $D^{b}\left(X_{\text {Zar }}, \mathbb{Z} / p^{r}\right)$

$$
\tau_{\leq n} R \epsilon_{*} \Im_{r}(n)_{X} \stackrel{\cong}{\longrightarrow} \tau_{\leq n}\left(\mathbb{Z}_{X}^{c} / p^{r}(d-n)^{Z a r}[-2 d]\right) .
$$

Recall that we use $\mathbb{Z}_{X}^{c} / p^{r}(d-n)^{\text {Zar }}$ to denote Bloch's complex of Zariski sheaves, and $\mathbb{Z}_{X}^{c} / p^{r}(d-n)$ to denote that of étale sheaves. Before proving the proposition, we prove the following lemmas: 
Lemma 4.6. Let $Z$ be a normal crossing variety over a perfect field of characteristic $p$ and $\operatorname{dim} Z=d-1$, then $H_{d+n-1}\left(\mathbb{Z}^{c} / p^{r}(n)\right)$ admites Gersten resolution, i.e., the following sequence of étale sheaves is exact:

$$
\begin{gathered}
0 \longrightarrow H_{d+n-1}\left(\mathbb{Z}_{Z}^{c} / p^{r}(n)\right) \longrightarrow \bigoplus_{Z_{(d-1)}} R^{1-d-n} i_{z *} \mathbb{Z}_{z}^{c} / p^{r}(n) \longrightarrow \ldots \\
\longrightarrow \bigoplus_{Z_{(n+1)}} R^{-2 n-1} i_{z *} \mathbb{Z}_{z}^{c} / p^{r}(n) \longrightarrow \bigoplus_{Z_{(n)}} R^{-2 n} i_{z *} \mathbb{Z}_{z}^{c} / p^{r}(n) \longrightarrow 0 .
\end{gathered}
$$

Proof. The above complex (without the first two terms) is $\left(C_{Z}^{H C}(n) / p^{r}\right)^{e} t$, the Gersten complex of $H_{d+n-1}\left(\mathbb{Z}_{Z}^{c} / p^{r}(n)\right)$. By Lemma 2.12 and Theorem 2.10, we know that $\left(C_{Z}^{\mathrm{HC}}(n) / p^{r}\right)^{\text {ét }}$ is isomorphic to $\widetilde{\nu}_{r, Z}(n)$. For normal crossing variety $Z$, the latter is quasiisomorphic to $\nu_{Z, r}^{d-n-1}[d+n-1]$ by Sato, [32, Corollary 2.5.2]. Hence, $\left(C_{Z}^{\mathrm{HC}}(n) / p^{r}\right)^{\text {ét }}$ is exact except at degree $1-d-n$. Consider the niveau spectral sequence of higher Chow groups in $\mathbb{Z} / p^{r}$-coefficients in (2.0.9), we see that the cohomology of $\left(C_{Z}^{\mathrm{HC}}(n) / p^{r}\right)^{\text {ét }}$ at degree $1-d-n$ is exactly $H_{d+n-1}\left(\mathbb{Z}_{Z}^{c} / p^{r}(n)\right)$.

Lemma 4.7. Let $F$ be a discrete valuation field of characteristic 0 with perfect residue field $k$ of characteristic $p$. Then the following square is commutative:

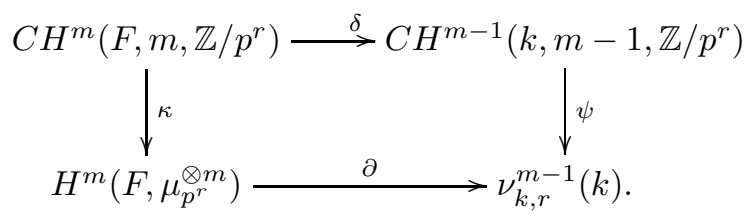

Here the map $\kappa$ is the map defined in [2], and $\delta$ is the defined in Section 2. The map $\psi$ is defined as the composition:

$$
C H^{m-1}\left(k, m-1, \mathbb{Z} / p^{r}\right) \longrightarrow K_{m-1}^{M}(k) / p^{r} \stackrel{d \log }{\cong} \nu_{k, r}^{m-1}(k),
$$

and $\partial$ is defined as the composition ([32], (3.2.3))

$$
H^{m}\left(F, \mu_{p^{r}}^{\otimes m}\right) \stackrel{\cong}{\mathfrak{g}} K_{m}^{M}(F) / p^{r} \stackrel{\partial^{\text {tame }}}{\longrightarrow} K_{m-1}^{M}(k) / p^{r} \stackrel{d \log }{\longrightarrow} \nu_{k, r}^{m-1}(k) .
$$

Here $\partial^{\text {tame }}$ is the tame symbol of Milnor K-theory, $d \log$ is the Bloch-Kato isomorphism, and $\mathfrak{g}$ is Galois symbol.

Proof. From the definition of the maps, the square in the lemma is actually formed from the following diagram

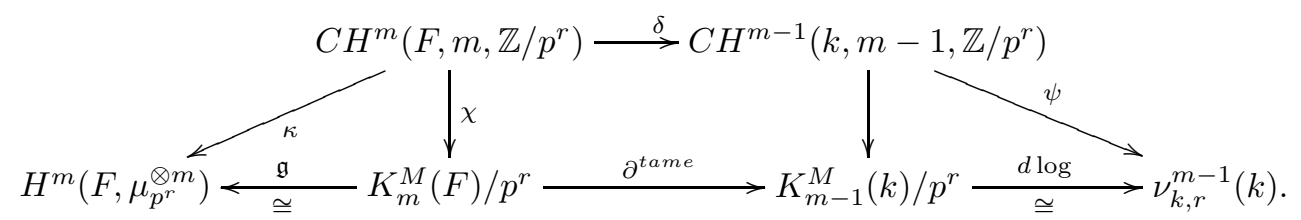

The map $\chi$ is the Nesterenko-Suslin isomorphism. So we have to show the trapezoid is commutative. The triangle on the right commutes by definition. The square in the middle commutes by Geisser-Levine, [14], Lemma 3.2. So we only have to analyze the triangle on the left. Since all the three maps $\kappa, \chi, \mathfrak{g}$ respect the products of higher Chow groups, 
Milnor K-theory and Galois cohomology, so it suffices to show that $\kappa=\mathfrak{g} \circ \chi$ for $m=1$. But in this case, all the three groups are identified with $F^{\times} / F^{\times p^{r}}$, and the three maps are just identity. Hence we proved the lemma.

Proof of Proposition 4.5. (1) $\Rightarrow$ (2): From Theorem 2.5, we see that $\tau_{\leq n}\left(\mathbb{Z}_{X}^{c} / p^{r}(d-n)^{Z a r}[-2 d]\right) \cong \tau_{\leq n} R \epsilon_{*}\left(\mathbb{Z}_{X}^{c} / p^{r}(d-n)[-2 d]\right) \cong \tau_{\leq n} R \epsilon_{*}\left(\tau_{\leq n} \mathbb{Z}_{X}^{c} / p^{r}(d-n)[-2 d]\right)$.

Hence, apply $\tau_{\leq n} R \epsilon_{*}$ on the isomorphism in (1), we obtain the one in (2).

(1) The idea of the proof is to show that $\mathbb{Z}_{X}^{c}(d-n) / p^{r}[-2 d]$ satisfies a triangle similar to the one for $\mathfrak{I}_{r}(n)_{X}$, and then show that there is a map of these two triangles which induces isomorphisms on the cohomologies up to degree $n$.

Let

$M_{X}=\mathbb{Z}_{X}^{c} / p^{r}(d-n)^{\mathrm{Zar}}[-2 d], M_{U}=\mathbb{Z}_{U}^{c} / p^{r}(d-n)^{\mathrm{Zar}}[-2 d], M_{Y}=\mathbb{Z}_{Y}^{c} / p^{r}(d-n)^{\mathrm{Zar}}[1-2 d]$.

Then by Theorem 2.1, there is a distinguish triangle of complexes of Zariski sheaves:

$$
M_{X} \rightarrow j_{*} M_{U} \stackrel{\delta}{\longrightarrow} \iota_{*} M_{Y}
$$

Since $\epsilon^{*}$ is exact and $\epsilon^{*} \mathbb{Z}_{X}^{c}(n)^{Z a r}=\mathbb{Z}_{X}^{c}(n)$, we get a distinguish triangle of complexes of etale sheaves

$$
\mathbb{Z}_{X}^{c} / p^{r}(d-n)[-2 d] \rightarrow \epsilon^{*} j_{*} M_{U} \stackrel{\delta}{\longrightarrow} \epsilon^{*} \iota_{*} M_{Y} .
$$

Consider the following diagram:

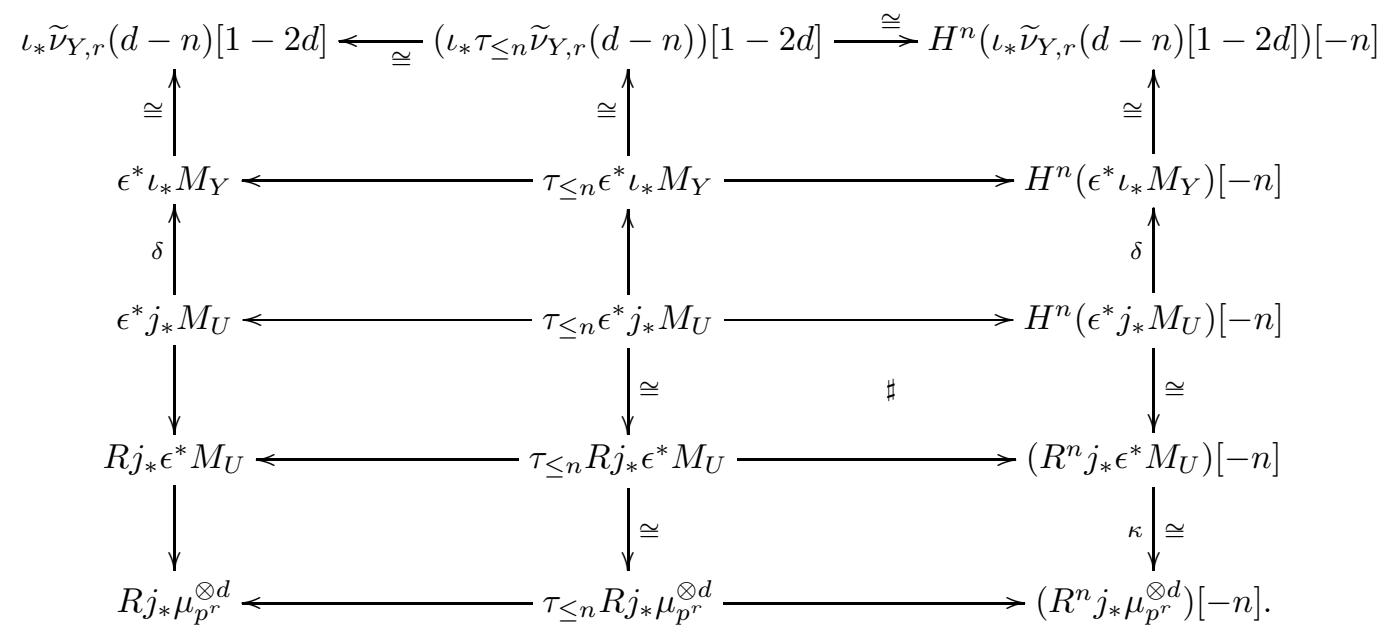

The maps on the left column are defined as follows: the first one is the map defined in Theorem 2.16, which is a quasi-isomorphism (note that $\epsilon^{*} \iota_{*}=\iota_{*} \epsilon^{*}$ ). The second map is the boundary map of localization sequence of higher Chow groups as above. The third map $\epsilon^{*} j_{*} \rightarrow R j_{*} \epsilon^{*}$ is induced by universal property of the étale sheafification $\epsilon^{*}$. The fourth map is defined in [23, §12]. The vertical maps in the second and third column are induced by taking truncation and cohomology of maps in the first column, respectively. Hence all the squares commute.

The maps in the first row are isomorphisms, since it is the Gersten resolution of $\nu_{Y, r}^{n-1}[-n]$ on normal crossing varieties over perfect fields of characteristic $p$ [33, Corollary 2.2.5(1)]. In particular, there is an isomorphism of sheaves

$$
\iota_{*} \nu_{Y, r}^{n-1} \stackrel{\cong}{\longrightarrow} H^{n}\left(\iota_{*} \widetilde{\nu}_{Y, r}(d-n)[1-2 d]\right),
$$


which, composing with the isomorphism

$$
H^{n}\left(\epsilon^{*} \iota_{*} M_{Y}\right) \stackrel{\cong}{\longrightarrow} H^{n}\left(\iota_{*} \widetilde{\nu}_{Y, r}(d-n)[1-2 d]\right),
$$

induces an isomorphism

$$
\psi: H^{n}\left(\epsilon^{*} \iota_{*} M_{Y}\right) \stackrel{\cong}{\longrightarrow} \iota_{*} \nu_{Y, r}^{n-1} .
$$

On the other hand, the Beilinson-Lichtenbaum Conjecture (Theorem 2.5) claims that

$$
\tau_{\leq n} R \epsilon_{*}\left(\epsilon^{*} M_{U}\right) \cong \tau_{\leq n} M_{U}
$$

Therefore,

$$
\tau_{\leq n} \epsilon^{*} j_{*} M_{U} \cong \tau_{\leq n} R j_{*} \epsilon^{*} M_{U}
$$

i.e., in the square $\sharp$, the vertical maps are isomorphisms. At last, the vertical maps in the lower right square are isomorphisms, by Theorem 2.6

In conclusion, we have the following diagram

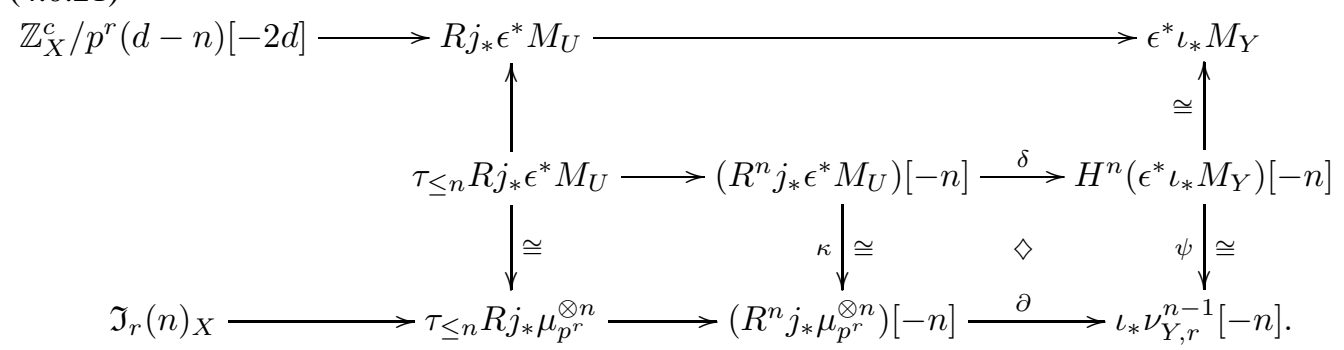

Here $\partial$ is the map $\sigma_{X, r}^{n}$ defined above. The top row and bottom row (with $\left(R^{n} j_{*} \mu_{p^{r}}^{\otimes n}\right)[-n]$ eliminated) are distinguished triangles. The upper square and lower left square commute as above. If we can show that the square $\diamond$ commutes, then it implies that there is a map

$$
\mathfrak{I}_{r}(n)_{X} \rightarrow \mathbb{Z}_{X}^{c} / p^{r}(d-n)[-2 d]
$$

which induces isomorphisms on cohomologies of $\mathbb{Z}_{X}^{c} / p^{r}(d-n)[-2 d]$ and $\mathfrak{I}_{r}(n)_{X}$ up to degree $n$, hence finishes the proof.

Now let us prove that the square $\diamond$ commutes. For $x \in U$, the right two terms vanish, hence the square $\diamond$ commutes. If $x \in Y$, we can take the henselization $S_{s}^{h}$ of $S$ at $s$, and prove the commutativity for all the irreducible components of $X_{s}=X \times_{S} S_{s}^{h}$ over $S_{s}^{h}$ (or replace $S_{s}^{h}$ by its integral closure in an irreducible component of $X_{s}$, depending on the Condition (4.1).

From now on, we assume that $A$ is a henselian discrete valuation ring with field of fraction $K$ of characteristic 0 and perfect residue field $k$ of characteristic $p$, and $X$ is flat, regular semistable family over $A$. Then $V=X_{K}$ is smooth over $K$ and $Z=X_{k} \stackrel{\iota}{\longrightarrow} X$ is a normal crossing variety over $k$. Since we are only left to show the commutativity of square $\diamond$ in the diagram 4.0.21 for points in $Z$, we can apply $\iota^{*}$ on the square, and obtain the following diagram:

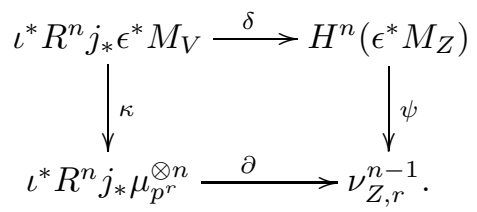

To show that this square commutes, we will use Gersten complex to replace all the maps by maps of functions fields of the schemes, and show compatibility of those maps. 
First, we have the following isomorphism of complexes:

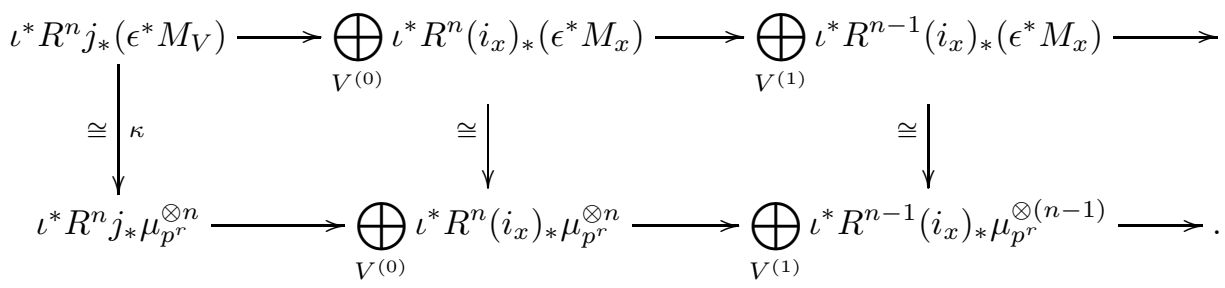

Here $i_{x}: x \rightarrow V \rightarrow X$ is the embedding into $X$, and $M_{x}=\mathbb{Z}_{x}^{c} / p^{r}(d-n)^{Z a r}[-2 i]$ if $x \in V_{(i)}$. Secondly, by [33], $\nu_{Z, r}^{n-1}$ on normal crossing variety admits Gersten resolution. By Lemma 4.6, higher Chow groups on normal crossing varieties admits Gersten resolution as well. More precisely, we have the following commutative diagram (2.16):

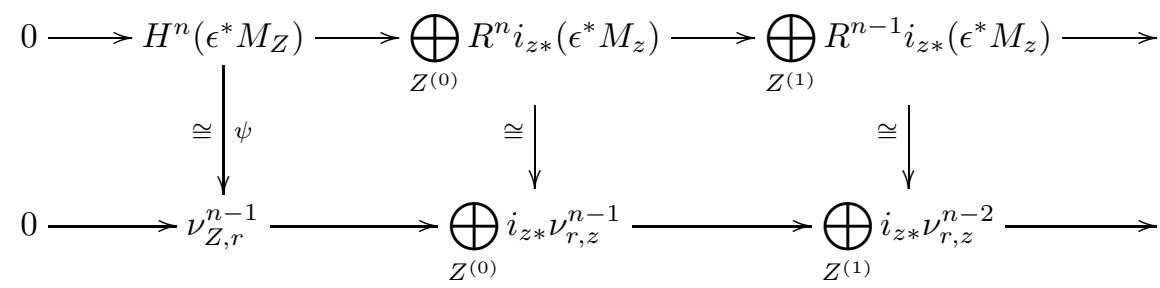

such that the rows are exact. Here $i_{z}: z \rightarrow Z$ are points in $Z, M_{z}=\mathbb{Z}_{z}^{c} / p^{r}(d-$ $n)^{Z a r}[-2 i-1]$ on $\operatorname{Spec} k(z)$ with $z \in Z^{(d-1-i)}=Z_{(i)}$. In particular, the map

$$
\nu_{Z, r}^{n-1} \rightarrow \bigoplus_{Z^{(0)}} i_{z *} \nu_{r, z}^{n-1}
$$

is injective. Thirdly, by functoriality of cycle complex, the following diagram is commutative,

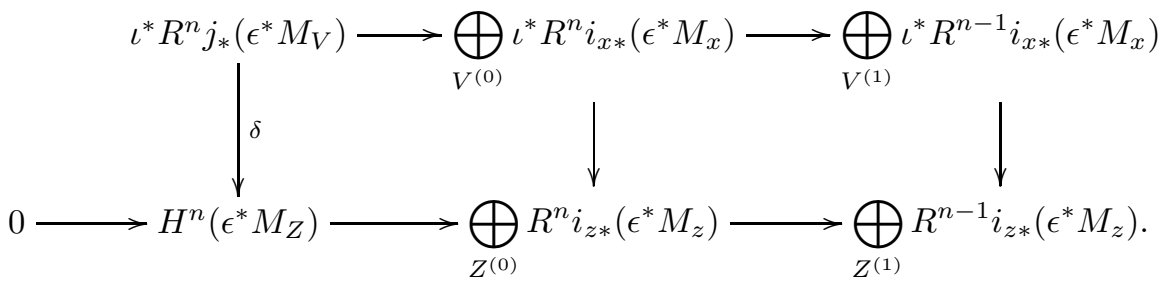

Finally, the map $\partial$ is induced by the diagram in 4.0 .19 .

To summarize the above analysis, let $z \in Z^{(0)}$. Replacing $X$ by the component containing $z$, we can assume that $X$ is irreducible, and $i_{\eta}: \eta \rightarrow X$ is the embedding of the generic point. To prove the commutativity of square (4.0.22), it suffices to prove that the following square commutes (here $i_{z}: z \rightarrow Z$ ):

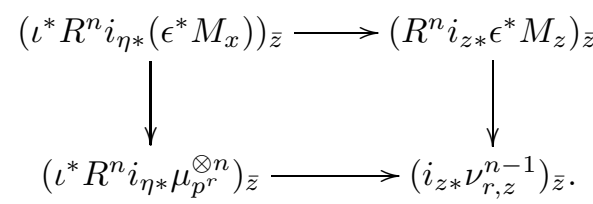

Since

$\left(\iota^{*} R^{n} i_{\eta *} \epsilon^{*} M_{x}\right)_{\bar{z}}=H_{e ́ t}^{n}\left(k\left(O_{X, \bar{z}}^{s h}\right), \mathbb{Z}^{c} / p^{r}(d-n)[-2 d]\right) \stackrel{\natural}{\cong} H_{2 d-n}^{\mathrm{BM}}\left(k\left(O_{X, \bar{z}}^{s h}\right), \mathbb{Z} / p^{r}(d-n)\right)$, 


$$
\begin{gathered}
\left(\iota^{*} R^{n} i_{\eta *} \mu_{p^{r}}^{\otimes n}\right)_{\bar{z}}=H_{e ́ t}^{n}\left(k\left(O_{X, \bar{z}}^{s h}\right), \mu_{p^{r}}^{\otimes n}\right), \\
\left(R^{n} i_{z *} \epsilon^{*} M_{z}\right)_{\bar{z}}=H_{\grave{e} t}^{n}\left(k(\bar{z}), \mathbb{Z}^{c} / p^{r}(d-n)[1-2 d]\right) \stackrel{\stackrel{\natural}{\cong}}{\cong} H_{2 d-n-1}^{\mathrm{BM}}\left(k(\bar{z}), \mathbb{Z}^{c} / p^{r}(d-n)\right), \\
\left(i_{z * *} \nu_{r, z}^{n-1}\right)_{\bar{z}}=\nu_{r, z}^{n-1}(k(\bar{z})) .
\end{gathered}
$$

Here $k\left(O_{X, \bar{z}}^{s h}\right)$ is the field of fraction of $O_{X, \bar{z}}^{s h}$, and the isomorphisms $\natural$ follow from the Beilinson-Lichtenbaum Conjecture (Theorem 2.5). Note that

$$
\begin{gathered}
H_{2 d-n}^{\mathrm{BM}}\left(k\left(O_{X, \bar{z}}^{s h}\right), \mathbb{Z} / p^{r}(d-n)\right)=C H^{n}\left(k\left(O_{X, \bar{z}}^{s h}\right), n, Z / p^{r}\right), \\
H_{2 d-n-1}^{\mathrm{BM}}\left(k(\bar{z}), \mathbb{Z}^{c} / p^{r}(d-n)\right)=C H^{n-1}\left(k(\bar{z}), n-1, \mathbb{Z} / p^{r}\right) .
\end{gathered}
$$

From Lemma4.7, we obtain the commutativity.

Theorem 4.8. For $X$ satisfying Sato's condition and $n \in[0, d]$, if the conjecture $\mathcal{B}(n)$ with $\mathbb{Z} / p^{r}$-coefficients holds for all the points in $X$, then

$$
\begin{gathered}
\mathfrak{I}_{r}(n)_{X} \stackrel{\cong}{\longrightarrow} \mathbb{Z}_{X}^{c} / p^{r}(d-n)[-2 d], \\
\tau_{\leq n} R \epsilon_{*} \Im_{r}(n)_{X} \stackrel{\cong}{\longrightarrow} \mathbb{Z}_{X}^{c} / p^{r}(d-n)^{\text {Zar }}[-2 d] .
\end{gathered}
$$

Proof. From Lemma 2.3, we see that the cycle complexes in the right hand side of the isomorphisms are acyclic at degree $>n$. Therefore, in the isomorphisms of Theorem 4.5 , the truncation in front of cycle complexes are not necessary.

\section{REFERENCES}

[1] Hyman Bass, John Tate, The Milnor ring of a global field, Algebraic K-theory II, Lecture Notes in Math. 342, Spring 1972, 349-466.

[2] Spencer Bloch, Algebraic cycles and higher K-theory, Adv. in Math. 61 (1986), No. 3, 267-304.

[3] Spencer Bloch, Arthur Ogus, Gersten Conjecture and the homology of schemes, Ann. Sci. Ec. Norm. Sup., 4. ser. 7 (1974), 181-202.

[4] Spencer Bloch, Kazuya Kato, p-adic étale cohomology, Inst. Hautes études Sci. Publ. Math. No. 63(1986), 107-152.

[5] Spencer Bloch, Stephen Lichtenbaum, A Spectral Sequence for Motivic Cohomology, K-theory Preprint, http://www.math.uiuc.edu/K-theory/0062/

[6] Jean-Louis Colliot-Thelene, Raymond Hoobler, Bruno Kahn, The Bloch-Ogus-Gabber theorem, Algebraic K-theory (Toronto, ON, 1996), 31 - 94.

[7] Christopher Deninger, Duality in the étale cohomology of one-dimensional proper schemes and generalizations, Math. Ann. 277 (1987), no. 3, 529-541.

[8] Kazuhiro Fujiwara, A proof of the absolute purity conjecture (after Gabber), Algebraic geometry 2000, Azumino (Hotaka), 153-183.

[9] Thomas Geisser, Duality via cycle complexes, Ann. of Math. Vol. 172(2010), No. 2, 1095-1126.

[10] Thomas Geisser, Motivic Cohomology over Dedekind Rings, Math. Z. 248 (2004), no. 4, 773-794.

[11] Thomas Geisser, Motivic Cohomology, K-theory and topological cyclic homology, Handbook of $K$-theory. Vol. 1, 2, 193-234, Springer, Berlin, 2005.

[12] Thomas Geisser, Parshin's conjecture revisited, $K$-theory and noncommutative geometry, 413-425, EMS Ser. Congr. Rep., Eur. Math. Soc., Zürich, 2008.

[13] Thomas Geisser, Marc Levine, The Bloch-Kato Conjecture and a theorem of Suslin-Voevodsky, J. Reine Angew. Math. 530 (2001), 55-103.

[14] Thomas Geisser, Marc Levine, The K-theory of fields in characteristic p, Inv. Math. 139 (2000), 459-494.

[15] Michel Gros, Noriyuki Suwa, La Conjecture de Gersten Pour Les Faisceaux de Hodge-Witt logarithmicique, Duke Math. J. 57 (1988), No. 2, 615-628.

[16] Uwe Jannsen, Shuji Saito, Kanetomo Sato, étale Duality for Constructible Sheaves on Arithmetic Schemes, K-theory Preprint, http://www.math.uiuc.edu/K-theory/0946/

[17] Bruno Kahn, Algebraic K-theory, Algebraic Cycles and Arithmetic Geometry, Handbook of $K$-theory. Vol. 1, 2, 351-428, Springer, Berlin, 2005.

[18] Kazuya Kato, A Generalization of Local Class Field Theory by Using K-groups II, J. Fac. Sci. Univ. Tokyo Sect. IA Math. 27(3), 603-683 (1980). 
[19] Kazuya Kato, Milnor K-theory and the Chow Group of Zero Cycles, Application of Algebraic K-theory to Algebraic Geometry and Number Theory, Contemp. Math. 55, Part I, Ameri. Math. Soc., Providence, 1986, 241-253.

[20] Kazuya Kato, A Hasse Principle for Two-dimensional Global Fields (with an appendix by Colliot-Thélène, J.-L.), J. Reine Angew. Math. 366 (1986) 142-183.

[21] Steven E. Landsburg, Relative Chow groups, Illinois J. Math. 35 (1991), No. 4, 618-641.

[22] Marc Levine, Techniques of Localization in the Theory of Algebraic Cycles, J. Alg. Geom. 10, 299-363 (2001).

[23] Marc Levine, K-theory and motivic cohomology of schemes, K-theory Preprint, http://www.math.uiuc.edu/K-theory/0336/

[24] Stephen Lichtenbaum, The Construction of Weight-two Arithmetic Cohomology, Invent. Math. 88 (1987), 183-215.

[25] James S. Milne, Etale cohomology, Princeton Math. Series 33.

[26] James S. Milne, Arithmetic Duality Theorems, Perspectives in Mathematics, 1. Academic Press, 1986.

[27] Thomas Moser, A Duality Theorem for étale p-Torsion Sheaves on Complete Varieties over a Finite Field, Composition Math. 117 (1999), No. 2, 123-152.

[28] Enric Nart, The Bloch Complex in Codimension One and Arithmetic Duality, J. Number Theory 32 (1989), no. 3, 321-331.

[29] Yu. P. Nesterenko, Andrei Suslin, Homology of the Full Linear Group over a Local Ring, and Milnor's K-theory, Izv. Akad. Nauk SSSR Ser. Mat. 53 (1989), no. 1, 121-146.

[30] Daniel Quillen, Algebraic K-theory, I: Higher K-theories, (Proc. Conf., Battelle Memorial Inst., Seattle, Wash., 1972), pp. 85-147. Lecture Notes in Math., Vol. 341, Springer, Berlin 1973.

[31] Markust Rost, Chow Groups with Coefficients, Doc. Math. 1(16), 319-393 (1996).

[32] Kanetomo Sato, p-adic Etale Tate Twists and Arithmetic Duality, With an appendix by Kei Hagihara. Ann. Sci. Ecole Norm. Sup. (4) 40 (2007), no. 4, 519-588.

[33] Kanetomo Sato, Logarithmic Hodeg-Witt Sheaves on normal crossing varieties, Math. Z. (2007) 257: 707743.

[34] Michael Spiess, Artin-Verdier Duality for Arithmetic Surfaces, Math. Ann. 305 (1996), No. 4, 705-792.

[35] Andrei Suslin, Vladimir Voevodsky, Bloch-Kato Conjecture and motivic cohomology, The arithmetic and geometry of algebraic cycles (Banff, AB, 1998), 117-189.

[36] Carlo Mazza, Vladimir Voevodsky, Charles Weibel,Lecture notes on motivic cohomology, Clay Mathematics Monographs, 2. American Mathematical Society, Providence, RI; Clay Mathematics Institute, Cambridge, MA, 2006.

Changlong Zhong

University of Southern California

Department of Mathematics,

3620 S Vermont Ave, KAP 108

Los Angeles, CA 90007

USA

Email: zhongusc@gmail.com 